This PDF is a selection from a published volume from the National Bureau of Economic Research

Volume Title: Economic Dimensions of Personalized and Precision Medicine

Volume Authors/Editors: Ernst R. Berndt, Dana P. Goldman, and John W. Rowe, editor

Volume Publisher: University of Chicago Press

Volume ISBNs: 978-0-226-61106-8 (cloth); 978-0-226-61123-5 (electronic)

Volume URL: http://www.nber.org/books/bern-13

Conference Date: September 13-14, 2017

Publication Date: April 2019

Chapter Title: Economic Dimensions of Personalized and Precision Medicine in Asia: Evidence from Breast Cancer Treatment in Taiwan

Chapter Author(s): Jui-fen Rachel Lu, Karen Eggleston, Joseph Tung-Chieh Chang

Chapter URL: http://www.nber.org/chapters/c13991

Chapter pages in book: (p. $237-272)$ 


\title{
Economic Dimensions of Personalized and Precision Medicine in Asia Evidence from Breast Cancer Treatment in Taiwan
}

\author{
Jui-fen Rachel Lu (盧瑞芬), Karen Eggleston (翁笙和), \\ and Joseph Tung-Chieh Chang (張東杰)
}

The economic and clinical factors that affect the growth of personalized and precision medicine (PPM) have universal features, but also vary across countries and institutional contexts. The economies of East Asia are interesting cases for understanding how recent rapid economic growth and population aging interacts with changing technologies of care. Moreover, Asia provides an interesting institutional setting for understanding the economic dimensions of PPM coverage expansions and the role of contractual arrangements for paying for companion diagnostic tests. One approach to expanding coverage in Asia has been to cover the PPM therapy, but require the pharmaceutical firm to cover the costs of the companion diagnostic test. Taiwan has embraced this approach for several cancers.

Jui-fen Rachel Lu is a professor in the Graduate Institute of Business and Management and Department of Health Care Management at Chang Gung University and a research fellow in the Department of Radiation Oncology at Chang Gung Memorial Hospital at Linkou. Karen Eggleston is director of the Asia Health Policy Program, deputy director of the Shorenstein Asia-Pacific Research Center, and senior fellow at the Freeman Spogli Institute for International Studies, all at Stanford University, and a faculty research fellow of the National Bureau of Economic Research. Joseph Tung-Chieh Chang is a professor and department chief and director of the Department of Radiation Oncology and the Proton Therapy and Radiation Therapy Center at Chang Gung Memorial Hospital.

The authors thank Ying Isabel Chen, Pin-Sung Peter Liu, Andrew Lee, and Christina Ping for excellent research assistance, and gratefully acknowledge funding to support this research from the Stanford Shorenstein Asia-Pacific Research Center (Shorenstein APARC faculty research award and Asia Health Policy Program), the National Bureau of Economic Research, Taiwan Ministry of Science and Technology (MOST 105-2410-H-182-017-MY2), and Chang Gung Memorial Hospital and Chang Gung University (BMRP285). This study is based in part on data from the Health and Welfare Data Science Center, Ministry of Health and Welfare (HWDC, MOHW). The interpretation and conclusions contained herein do not represent those of Ministry of Health and Welfare. The authors are solely responsible for views presented in this chapter. For acknowledgments, sources of research support, and disclosure of the authors' material financial relationships, if any, please see http://www.nber.org/chapters/c13991.ack. 
Such arrangements for protecting patients from the high costs associated with precision medicine treatment are important because otherwise the introduction and spread of PPM might exacerbate any existing incomerelated disparities in health care utilization and outcomes. Lack of knowledge and the costs of diagnostic testing and treatment are among the factors contributing to such disparities. However, to the extent that lower-income populations suffer disproportionately from an indication covered by precision medicine, such patients may also disproportionately benefit from the new technology and its diffusion in clinical practice. For example, if poorer patients with cancer tend to present with later stages of the disease, they may disproportionately benefit from precision medicine targeted to latestage and/or metastatic cancer. We test this hypothesis for breast cancer as a case combining the two primary factors mentioned: propensity for poorest patients to be diagnosed at later stages, and among the first approved targeted treatments.

This study is the first in a series that examines the Taiwan experience over the past decade with incorporating PPM into National Health Insurance (NHI) coverage, and its implications on disparities in treatment. Taiwan, as a prototypical "Asian tiger," has a system straining to finance universal health coverage under pressures of rising population expectations and the ever-increasing capabilities of medicine. The economics of Taiwan's coverage expansions present an interesting case, in part because Taiwan's NHI covers target therapies under an agreement that the cost of genetic diagnostic testing for lung cancer, colorectal cancer, and leukemia will be paid by the pharmaceutical firm supplying the treatment.

However, the first target therapy NHI covered did not feature this contractual arrangement with pharmaceutical firms. The NHI covered trastuzumab (Herceptin) for human epidermal growth factor receptor type 2 (HER2)-positive metastatic breast cancer without any agreement on who would pay for companion diagnostic tests. Although systematic data is not available, interviews and clinical experience suggest that prior to NHI coverage, genomic tumor testing costs for breast cancer were paid by the patient out of pocket, or occasionally by the provider's medical research fund on a case-by-case basis. The prevalence of pharmaceutical company coverage of testing and its impact on patient access are topics of our ongoing research.

Studies in many countries, including the United States, have shown the impact of coverage policies on disparities in breast cancer screening, stage at diagnosis, and treatment (e.g., Silva et al. 2017; Tarazi et al. 2017). For example, research on Medicare claims data by Layne et al. showed that breast cancer patients in US territories (such as Guam and Puerto Rico) experienced a marked delay and poorer access to many standard treatments for breast cancer, as compared to their counterparts in the continental United States, who generally have higher income (Layne et al. 2018). In China, Wang et al. 
(2012) found that 26 percent of women were diagnosed with Stage III or IV breast cancer in low socioeconomic status (SES) regions, like Sichuan, compared to 15 percent in high SES regions, like Beijing. Conversely, more women in high SES regions were diagnosed with early stage breast cancer (28 percent), compared to 11 percent in low SES regions. Women in rural areas tend to receive later diagnoses (stage $>3$ ) than in urban areas. Chang et al. (2012) found a similar pattern in Taiwan, although there were issues with how they used the data to measure SES.

Our study focuses on breast cancer treatment as a case study in PPM expansion and whether PPM coverage is pro-poor or pro-rich in its utilization patterns. Breast cancer is a leading cause of cancer-related deaths worldwide among women. In 2012, there were 1.7 million new cases of female breast cancer globally, with an age-adjusted incidence rate of 47 per 100,000 women (WHO 2012). In Asia, it is the second leading cause of cancer-related deaths among women, accounting for 39 percent of all breast cancers diagnosed worldwide (Fan, Goss, and Strasser-Weippl 2015). The incidence of breast cancer in Asia is 27 per 100,000, but varies widely across the continent. Moreover, the proportional contribution of Asia to global breast cancer has increased rapidly (WHO 2012) and the mortality-to-incidence ratios have been higher in Asia than in Western countries, potentially because of lack of access to the latest effective treatments.

HER2 positivity accounts for almost one in five breast cancers, and a higher proportion - a little more than one in four breast cancers - among ethnically Chinese women ( $\mathrm{Li}$ et al. 2011). Target therapy has transformed treatment. As one recent clinical review noted, "anti-HER2 treatment ... . has changed the natural biology of this disease ... and clearly improved the prognosis of HER2-positive breast cancer" (Loibl and Gianni 2017), although it can be very expensive. Thus, providing access to anti-HER2 therapy is important for a Chinese population with growing incidence and prevalence of breast cancer, such as Taiwan.

The breast cancer incidence among females was 69.1 per 100,000 in 2013 in Taiwan, almost threefold greater than the reported incidence in Asia, consistent with its higher income and rapid economic transition. Ethnically Chinese breast cancer patients present at an earlier age (cluster peak from forty to forty-nine years old versus sixty to sixty-nine years old) than US women, and also with a higher prevalence of HER2-positive tumors (25.8 percent versus 15 percent; Li et al. [2011]). As one of the only cases of PPM covered for many years by the National Health Insurance Administration in Taiwan and other parts of developed Asia, the case of breast cancer treatment can elucidate how increasingly generous coverage of PPM, including coverage of the companion diagnostic test, can benefit lowerincome patients. Policymakers clearly are concerned with controlling costs to assure access as well as improve quality, as manifested by a promising 
pilot program for bundled payment of breast cancer treatment (Wang, Cheng, and $\mathrm{Wu} 2017){ }^{1}$

Using a unique, nationally representative data set for breast cancer treatment and survival, we examine the roll-out and diffusion of this case of PPM, and analyze trends in disparities with a concentration index. The medical utilization for diagnostic tests and treatment therapies from NHI claims data can be linked to the cancer registry by using the individual's national ID created by the Ministry of Health and Welfare (MOHW) Health and Welfare Data Science Centre. We explore if there is income-related inequality in receiving genetic testing and target therapy for breast cancer under Taiwan NHI, and whether increasing coverage over time made the utilization more pro-poor. In Taiwan, pharmaceutical companies often cover the cost of diagnostic genomic testing, insulating poor patients from out-ofpocket expenses that might limit their access to testing and thus treatment. While no systematic data on the prevalence of firms' coverage of tumor testing is available for Taiwan, we can observe the extent to which cancer patients of different income levels accessed target therapy and thus deduce whether NHI coverage of testing made target therapy utilization more pro-poor.

To guide our hypotheses and interpretation, we utilize a simple model showing that the overall expected value of target therapy does not have an unambiguous monotonic relationship with SES. Poorer patients benefit more to the extent that they are more likely to be at later stage at diagnosis, but richer patients benefit more to the extent that they have higher willingness and ability to pay for target therapy, especially the combination target therapy of trastuzumab with pertuzumab that is never fully covered by NHI. Thus, a priori it is unclear whether the concentration index for trastuzumab use will be positive or negative. We hypothesize that insurance coverage for this example of PPM may be pro-poor, given that coverage cannot erase disparities manifest in the form of poorer patients presenting at later stages of the disease, the stages for which target treatment was first developed and covered. We find empirical support for this conjecture.

The remainder of the chapter is organized as follows. First, we provide background information on coverage of personalized medicine in Asia, an overview of Taiwan's health system, the epidemiology of breast cancer in Taiwan, and Taiwan NHI policies surrounding testing for HER2-positive

1. We do not currently have access to information on which patients in our data may have participated in this bundled-payment scheme since it is not crucial for the hypotheses tested in this study; we will include such data in follow-on research about resource use and supply-side incentives. According to the NHIA, only four hospitals participate in the breast cancer bundledpayment scheme, two public hospitals and two foundation-owned hospitals. The breast cancer bundled-payment scheme is mainly for inpatient services and there are quite a few "bundles" (i.e., different combinations of surgery, chemotherapy, and other treatments). In general, if trastuzumab is given during the hospitalization, then it is covered in the bundled payment. 
breast cancer and its treatment. Then we articulate our hypotheses with a simple model, and introduce our data and empirical methods. The summary of results is followed by a brief discussion and conclusion.

\subsection{Background}

\subsubsection{Coverage of Personalized Medicine in Asia}

Study of approval and coverage of personalized medicine in Asia can contribute to the understanding of the trade-offs made in practice as such technologies diffuse in diverse parts of the world. Interviews we conducted in Taiwan, as well as South Korea, China, and Japan, reveal that similar issues are salient to purchasers in East Asia as to purchasers elsewhere, including health plans in the United States: namely, efficacy and cost of the test, number of patients affected, and the extent to which the test results guide clinical treatment (Appold 2017; Pauly 2017). The coverage decisions for personalized therapies and their diagnostic tests usually proceed through the same steps of approval as for other tests, medications, and devices. Following this logic, coverage for cancer tumor testing has generally been incorporated into standard insurance coverage in each country before coverage for other PPM, such as hereditary cancer predisposition testing (which to date is not covered in Japan; e.g., Asano 2017). Trastuzumab (Herceptin) for breast cancer was among the very first precision therapies covered, starting in 2001 in Japan and 2002 in Taiwan (i.e., NHI covered trastuzumab treatment of metastatic breast cancer starting in 2002, prior to our study period). Indeed, in more recent years and for high-prevalence cancers, approvals occasionally occur in Asia first. For example, gefitinib (Iressa; for lung cancer) was approved in Japan in 2002, prior to its approval in the United States and the European Union (Asano 2017).

None of the East Asian countries studied explicitly incorporate cost effectiveness into national insurance coverage decisions like the National Institute for Health and Care Excellence (NICE) in the United Kingdom, but they all have a staged approval process that balances clinical benefits for patients with the realities of the budget process. Even in high-income Australia, the government created a separate "Herceptin Program" in 2001 to provide access for breast cancer patients because a cost-effectiveness analysis that year led to rejection of Herceptin for coverage (Neumann et al. 2017,12 ). The affordability of precision medicines is unsurprisingly a more stringent constraint for low- and middle-income countries, leading to large disparities in clinical areas most affected by PPM, like cancer diagnosis and treatment. Testing is sometimes covered before treatment, given the high costs of the latter, as was the case in China for HER2-positive breast cancer before 2017. Although the testing had been covered by most insurance programs earlier, and Roche started a patient assistance program in 
$2011,{ }^{2}$ until recently only around half of breast cancer patients in China were tested and only about 30 percent of HER $2+$ patients actually use trastuzumab for treatment (Hicks, Liu, and Zhao 2011). Trastuzumab was added to the insurance reimbursement list in July 2017 along with several other leading cancer drugs as part of a negotiated reduced-price agreement between pharmaceutical firms and China's Ministry of Human Resources and Social Security (Jourdan 2017).

There is also an interplay between coverage decisions and development of the biotech industry in the region. China launched a precision medicine initiative in 2016 with over US\$9 billion in funding, and other countries in the region also aim to become global leaders in the field. More specifically, regarding breast cancer treatments, for example, the first biosimilar to Herceptin, developed by Biocon and Mylan, received market authorization in India in 2013. The Korean Ministry of Food and Drug Safety approved coverage for biosimilar Herzuma in 2014, produced by the South Korean biotech company Celltrion; commercialization was delayed by a Roche patent infringement lawsuit, but Seoul Central District Court ruled in favor of the Korean firm in April 2017 (Sohn 2016, 2017). Other firms in the region also aim to enter the breast cancer market; the Korean firm Alteogen, for example, is contracting with a Chinese firm, Qilu, in developing another Herceptin biosimilar. ${ }^{3}$

Similar to many other policy authorities in the region and globally, the Taiwan National Health Insurance Administration (NHIA) confronts some controversies in setting its reimbursement policy for precision medicinesto date, predominantly in oncology. At this point, Taiwan NHIA only reimburses a limited number of target therapies for cancer patients for two primary reasons: first, these treatment regimens are high cost, generally without sufficient evidence (within an ethnically Chinese patient population) to prove effectiveness and cost effectiveness. Second, institutional barriers limit scale-up because of questions about the validity and reliability of the diagnostic tests and the lack of Taiwan Food and Drug Administration (TFDA)-certified labs to conduct the tests. The Taiwan NHIA is looking for evidence to validate the effectiveness and cost effectiveness of PPM diagnostic tests and treatment regimens.

\subsubsection{Taiwan's Health System}

In 2015, Taiwan National Health Insurance (NHI) celebrated its twentieth anniversary since its historic inauguration in 1995. The NHI program,

2. "In collaboration with the Cancer Foundation in China and the Ministry of Health, we launched a patient assistance program (PAP) in August 2011 to address affordability. Under the program, after a patient has taken the first six cycles of Herceptin treatment, Roche donates the next eight cycles through the Cancer Foundation so that patients complete the full course of treatment." http://www.roche.com/sustainability/what_we_do/for_patients/access_to _healthcare/making_innovation_accessible/ath_china_pap.htm.

3. The Investor, Mar. 30, 2017. http://www.theinvestor.co.kr/view.php?ud=20170330000590. 
which provides universal health coverage (UHC) to Taiwan's population of 23 million, has had a profound impact on Taiwan's health care market. The single-payer NHI program, operated by the National Health Insurance Administration (formerly known as Bureau of NHI), was established through integrating three existing social insurance schemes and extending coverage to the remaining 43 percent of the population who had been uninsured. Taiwan NHI offers comprehensive benefit coverage that includes ambulatory care as well as inpatient services. On the service side, Taiwan has a market-oriented health care delivery system, reflecting its free-enterprise economy, as evidenced by the pluralistic organization of health services. Hospital ownership is mixed, with public hospitals accounting for 35 percent of all beds. Sixty-three percent of allopathic physicians are salaried employees of hospitals; the remainder are fee-for-service private practitioners. Over the years, hospitals have developed large outpatient departments and affiliated clinics for primary care to maintain inpatient volume and compete with private practitioners who operate freestanding clinics with beds. There is no gate-keeping mechanism and the insured essentially enjoy complete freedom of choice, which is likely a source of overuse (Lu and Hsiao 2003; Lu and Chiang 2011).

The NHI revenue mainly relies on payroll-based premiums, supplemented by a levy on nonpayroll income and government subsidies. In 2015, NHI spent roughly NTD 543.57 (USD 18.12) billion on medical claims, accounting for 52.81 percent of national health expenditures, and in total, Taiwan devoted 6.14 percent of gross domestic product (GDP) to health (Ministry of Health and Welfare 2017). As a single payer, NHIA has effectively exploited its market power to experiment with various payment reforms in its twenty-two-year history. The NHIA gradually set up separate global budgets for dental services, Chinese medicines, primary care services, and hospital services since 1998. The annual growth rate of the total NHI budget is negotiated among stakeholders in the previous year.

\subsubsection{Breast Cancer and Its Treatment in Taiwan}

In Taiwan, female breast cancer incidence has increased significantly over the past thirty years for all age groups over eighteen. From 1980 to 2010, the mean incidence of breast cancer increased almost seven times (from 11.40 to 73.27 per 100,000 ), with a 50 percent increase in the last decade. The forty-five to sixty-four age group experienced a threefold increase since 1981 (TCR 2013).

According to the Taiwan Cancer Registry, incidence peaked at 164 per 100,000 among the forty to sixty age cohort, compared to Western countries where the peak incidence tends to occur among older cohorts (age $>60$ ) (TCR 2013; DeSantis et al. 2016). This reflects a younger age of tumor onset (Shen et al. 2005). More than 50 percent of patients diagnosed with breast cancers in Asian countries are premenopausal, a proportion nearly twice that of Western countries. Similarly, the incidence ratio between younger 
(age $<50$ ) and older patients (age $>50$ ) with breast cancer is 0.55 , which is also double that of Western countries (0.26) (Parkins, Pisani, and Ferlay 1993; Huang et al. 2010).

In 2010, the breast cancer age-standardized mortality rate in Taiwan was 18.1 per 100,000 . Breast cancer mortality rates have increased more than twofold from 1971 to 2010 . There was a 55 percent increase in mortality for the twenty to forty-four age group, and a 150 percent increase for the fortyfive to sixty-four age group (Ho et al. 2015), at the same time that mortality was decreasing in western countries. The one-year survival rate for all breast cancer stages in females was 97.3 percent, and the five-year survival rate was 83 percent, which is comparable to five-year survival in the United States and Europe (TCR 2013).

With NHI providing 99 percent coverage since 2004, cancer care is almost universally accessible to patients in Taiwan. Biennial breast cancer screening and mammography have been available without charge to patients as well. Pan et al. (2014) found that in 2011 and 2012, the biennial mammography coverage rate was 33.2 percent. Increasing resources have been devoted to screening, including adoption of digital mammography, mobile mammography units, and the certification of radiologists and radiographers. As these resources grow, the coverage rate is expected to grow as well (Pan et al. 2014). The age forty to forty-nine cohort had the highest rate of mammography, breast ultrasound, and physician examination, corresponding with the recommended age for Asian women to begin breast cancer screening (Lin 2008; Tsuchida et al. 2015). Despite the increase in screening utilization over the past decade, disparities still exist. For example, high school graduates were half as likely to receive a mammogram or breast ultrasound as college graduates (Lin 2008). In addition to disparities in knowledge, awareness, and prevention, those living in more remote areas of the island might also face geographic constraints in accessing treatment. As Einav, Finkelstein, and Williams (2016) highlight, even in the United States and other high-income countries, distance to the nearest provider for repeated treatments such as radiation therapy can significantly shape treatment decisions.

The NHIA requires gene testing for all cancer target therapy. During our study period, the only gene expression tests for target therapy that were covered by NHI were the ImmunoHistoChemistry (IHC) since 2004 and the fluorescence in situ hybridization (FISH) since 2009. Breast cancer target therapy will only be covered if IHC tested 3+ or FISH tested positive (figure 9.1); the FISH test might be performed if the IHC test was suggestive but not definitive, such as $2+$. In contrast, as noted above, for other cancers including lung cancer, colon cancer, and leukemia, companion diagnostic tests are generally paid for by the pharmaceutical firm supplying the treatment, sometimes supplemented by the patient's own out-of-pocket payments or by the provider's medical research fund. 


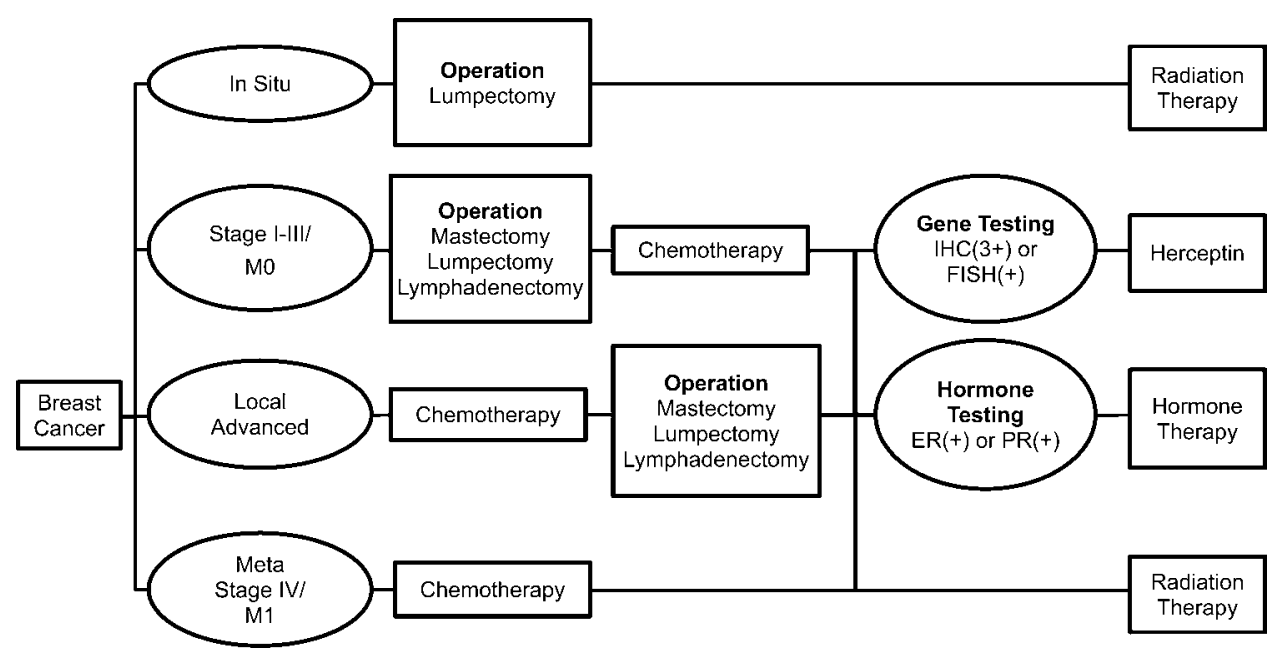

Fig. 9.1 Breast cancer treatment regimen

In our empirical analyses, we examine whether the cost of genetic testing potentially could be an access barrier to target therapy for breast cancer patients by utilizing the natural experiment of 2009 coverage of the FISH test, as well as the 2010 coverage of trastuzumab treatment for early stage breast cancer.

Our study is constrained by the fact that there will be no data in NHI claims if the testing or treatment is not covered by NHI. Accordingly, we are likely to underestimate actual utilization of target therapy among the highest-income patients, who are those most likely to pay for such treatment out of their pockets. Even though NHI covered trastuzumab, pertuzumab is not covered by NHI, so we have no data about whether higher-income patients were more likely to utilize the combination target therapy of the two (a likely case, given that the latter adds additional treatment efficacy). In addition, per NHI guidelines, one needs a positive testing result before target therapy treatment can be covered; however, we do not observe the test results, only the prescription of the test. So we are testing whether the probability of targeted treatment will less significantly differ with income quintile after NHI expands coverage - first, for FISH testing, then for early stage cancer (i.e., if utilization will be more pro-poor than it was before the coverage of the test). The NHI coverage for both the companion diagnostic and the treatment may erase income-related inequalities (disparities) in utilization of targeted therapy for a given stage of cancer, and disproportionately benefit poorer patients who present with the later stage cancer. The next section presents our hypotheses with greater precision, using a simple model. 


\subsection{Conceptual Framework}

Let $h$ represent the probability that an individual with breast cancer has the HER2-positive molecular subtype of breast cancer and thus would benefit from anti-HER 2 target therapy. We study this case in part because we know $h$ is relatively large for ethnically Chinese women (25.8 percent prevalence of HER2-positive tumors, compared to 15 percent in the general US population; Li et al. [2011]). We assume that all Taiwanese have the same basic genetic propensity and therefore that $h$ is constant, independent of income $y$ or broader measures of SES. The expected value of target therapy, EV, depends on the individual's clinical appropriateness, as well as willingness and ability to pay for the treatment, given NHI coverage policies. For example, prior to 2009, coverage was conditional on having metastatic HER2-positive breast cancer. In other words, any woman with early stage cancer, or late stage cancer that was not yet metastatic, could not have trastuzumab covered by NHI and instead had to pay for the treatment themselves. Let $\omega(y)$ represent the stage of breast cancer at diagnosis. Unlike $h$, we hypothesize this probability will be a function of income, because the likelihood that breast cancer is detected at an early stage is likely to be increasing with income: $\omega^{\prime}(y)<0$. We see this tendency for higher SES patients to receive diagnosis and treatment at earlier stages of cancer in many parts of the world. ${ }^{4}$

НyPOTHESIS 1: The likelihood that a given patient will have more advanced (later stage) breast cancer at diagnosis is decreasing in income: $\omega^{\prime}(y)<0$.

Let $V(m ; y, \theta)$ represent patient utility from treatment, where $m$ represents spending (resource use) for target therapy, and $\theta$ is the patient co-insurance rate for services covered by insurance $(0 \leq \theta<1$, equal to 0 when fully covered by NHI).

The expected value of NHI coverage for target therapy depends on the probability of a patient having HER2-positive breast cancer, the stage at diagnosis, and the demand for target therapy given NIH coverage policies

$$
E V(m)=h \omega(y) V(m ; y, \theta) .
$$

Although the rich and poor are equally likely to be HER2-positive, they do not enjoy the same EV. The overall expected value of target therapy does not have an unambiguous monotonic relationship with SES. On the

4. In addition to the sources cited in the introduction, see, for example, Daly and Olopade (2015) and sources cited therein highlighting that in the United States, African American women present at more advanced stages of breast cancer. As noted, in Taiwan, high school graduates were half as likely to receive a mammogram or breast ultrasound as college graduates (Lin 2008). Similarly, in Hong Kong, Chan et al. (2002) found women with lower education were less likely to receive a clinical breast examination or to perform a breast self-examination, compared to women with higher educational attainment. 
one hand, poorer patients benefit more from NHI coverage of trastuzumab to the extent that they are more likely to be at a later stage at diagnosis; especially prior to 2010 , poorer patients would be expected to benefit from trastuzumab coverage if, as Hypothesis 1 surmises, they are more likely to be metastatic at diagnosis (M1) or to develop metastasis during the study period (i.e., because they are at a later stage at initial diagnosis). In other words, the first term $h \omega(y)$ is higher for low-income patients. However, the second term $V(m ; y, \theta)$ is higher for richer patients to the extent that they have higher willingness and ability to pay for target therapy, especially the combination target therapy that is never fully covered by NHI. Thus, a priori it is unclear whether the concentration index for trastuzumab use will be positive or negative; it is an empirical question we address in this study.

HypотHESIs 2: The overall association between patient income and receipt of trastuzumab may be positive or negative; the greater the propensity for low-income patients to present with later stage disease, the more likely that target therapy utilization is pro-poor (i.e., with a negative concentration index), especially prior to the 2010 extension of coverage to patients with early stage breast cancer.

The NHI coverage of trastuzumab and the IHC companion diagnostic test-but not of complementary target therapy such as pertuzumab (or before 2009, the FISH companion diagnostic test) — can be considered a kind of "top-up" insurance policy with potential efficiency properties, depending on ex ante risk (Einav, Finkelstein, and Williams 2016). Taiwan's coverage before 2009 was equivalent to a deductible for the FISH diagnostic test, and then $\theta=0$ if the patient had HER2-positive metastatic breast cancer and did not also use pertuzumab. In 2009, this deductible was removed (the FISH test was fully covered) for metastatic cases.

HyPOTHESIS 3: The proportion of lower-income patients receiving target therapy increases after 2009 when the more expensive of the companion diagnostic tests, the FISH test, is covered by insurance.

Following on the heels of the FISH policy change, in 2010 the NHI removed the requirement of having metastatic cancer in order to qualify for trastuzumab treatment. In other words, trastuzumab was reimbursed for patients with early stage breast cancer starting in 2010. The requirement to self-pay for pertuzumab therapy - that is, a substantial co-insurance requirement $(\theta>0)$ for combined trastuzumab + pertuzumab target therapy ${ }^{5}$ - continued throughout our study period, so higher-income patients might be more likely to be able to "top-up" to utilize the most effective target therapy combination for HER2-positive breast cancer. Unfortunately, with the NHI data in this study, we cannot observe the use of any services not covered by

5. A course of pertuzumab treatment could cost over US\$60,000. 
NHI; thus, we do not know which patients received the combined target therapy by paying out of pocket for pertuzumab. However, we do observe survival, which may provide some insight into which patients had access to the combined target therapy that extends survival.

\subsection{Data and Empirical Methods}

\subsubsection{Data Description}

The data files linked to construct the study sample include the cancer registry, death registry, and NHI claim files (including both inpatient and outpatient services). Recognizing the rising trend in cancer incidence, the Taiwan Department of Health ([DOH]; now Ministry of Health and Welfare [MOHW]) launched the Taiwan Cancer Registry (TCR) in 1979 to monitor cancer incidence. Since then, the TCR central office has collected basic information (short-form database, twenty items, now forty-two items) on newly diagnosed malignant cancer patients from hospitals with more than fifty beds throughout Taiwan. Starting in 2002, the scope of data expanded to include more detailed information such as cancer staging, first course of treatment, and follow-up data (long-form database, sixty-five items, now 114 items). With the enactment of the Cancer Control Act in 2003, DOH made the reporting process mandatory for all medical institutions and launched a trace-back procedure to enhance the quality of the cancer registry. To date, the long-form database accounts for more than 90 percent of total cancer cases in Taiwan (Chiang et al. 2015). The quality of the cancer registry has been validated by indicators such as morphologically verified cases (MV percent), the mortality versus incidence ratio (M/I percent) and the percentage of death-certificate-only cases from 1980-1984 to 2000-2006; each of these measures has shown steady improvement (Chiang 2010). Breast cancer is one of the six cancers with mandatory reporting since 2004; our analytical sample is hence restricted to newly diagnosed breast cancer cases identified in 2004 and later. While we cannot rule out differential coverage by income or other socioeconomic factors, the 90 percent coverage rate of the cancer registry by 2015 provides some reassurance.

Taiwan implemented its NHI program in 1995 and has released the claims data and registration files for research use via the National Health Research Institutes (NHRI) since 2000. In the face of rising public concern over patient confidentiality, since $2015 \mathrm{MOHW}$ has restricted access to NHI claims data to the Health and Welfare Data Science Centers, one of which we used for this study. We linked the cancer registry data to NHI claims data that includes all insurance-covered utilization, including prescription and other medications, outpatient and inpatient services, and the characteristics of the provider (e.g., physician specialty, clinic or hospital and its ownership). To examine whether patterns of use differ by geographic region, we 
code each patient to one of six NHI division offices, as well as utilize an official designation of geographically remote areas (based on the MOHW definition). To obtain information on monthly insured wage and residency proxy, we have also used the registry of beneficiaries (underwriting) file.

Figure 9.2 depicts the sample construction process. We identified newly diagnosed breast cancer patients (ICD-O-3 beginning with $\mathrm{C} 50 . \mathrm{XX}$ ) from the 2004-2013 TCR long-form database $(N=86,618)$ and then linked those records with 2004-2015 NHI claims data, as well as the registry of beneficiaries and death registry. The claims data provides information on treatment and medication as well as some SES variables (location of NHI enrollment and, for some enrollees, insured monthly wage), to be discussed in more detail below.

Cancer staging is crucial for our analyses, but we discovered that there are a nontrivial number of missing values for staging in the cancer registry data. Breast cancer is staged using the American Joint Committee on Cancer (AJCC) TNM system, which is based on three key pieces of information: the size of the original tumor $(T)$ and if it has grown into nearby areas, whether the cancer has reached nearby lymph nodes $(N)$, and whether the cancer has metastasized (spread to other parts of the body) (M). The M staging variable is missing for 6.8 percent of our analytical sample. The TCR also codes the overall stage (Health Promotion Administration 2016).

To link up the data from the TCR, NHI claims and registration, and death registry, all the analyses were conducted at the Health and Welfare Data Science Center at Chang Gung University branch site. We obtained institutional review board (IRB) approval for this study from the Research Ethics Office of National Taiwan University.

Despite the comprehensive information regarding utilization embedded in the NHI database, a drawback is its lack of socioeconomic information, such as educational attainment, household income, and residency (the NHI data also do not include any information about the testing results or any services that were paid for entirely out of pocket).

To study whether coverage mitigated disparities in utilization, we developed two strategies to measure the economic status of breast cancer patients. One is to use the monthly insured wage in the Registry of Beneficiaries file. While this data is appealing because it is accurate at the individual level, it is only available for a specific subgroup of the population (Category I insured); namely, people who are also insured by Labor Insurance (workers in the formal sector) and Government Employee Insurance (government employees and faculty members in private schools and universities; Lien [2011]). As the lowest monthly insured wage is the legal minimum wage, we have deleted observations with reported insured wage less than the minimum wage. Our second method is to use the district-specific median household income for all 368 districts from the tax return data released by the Ministry of Finance. We used median income data for year 2012, as it was the first year when the 


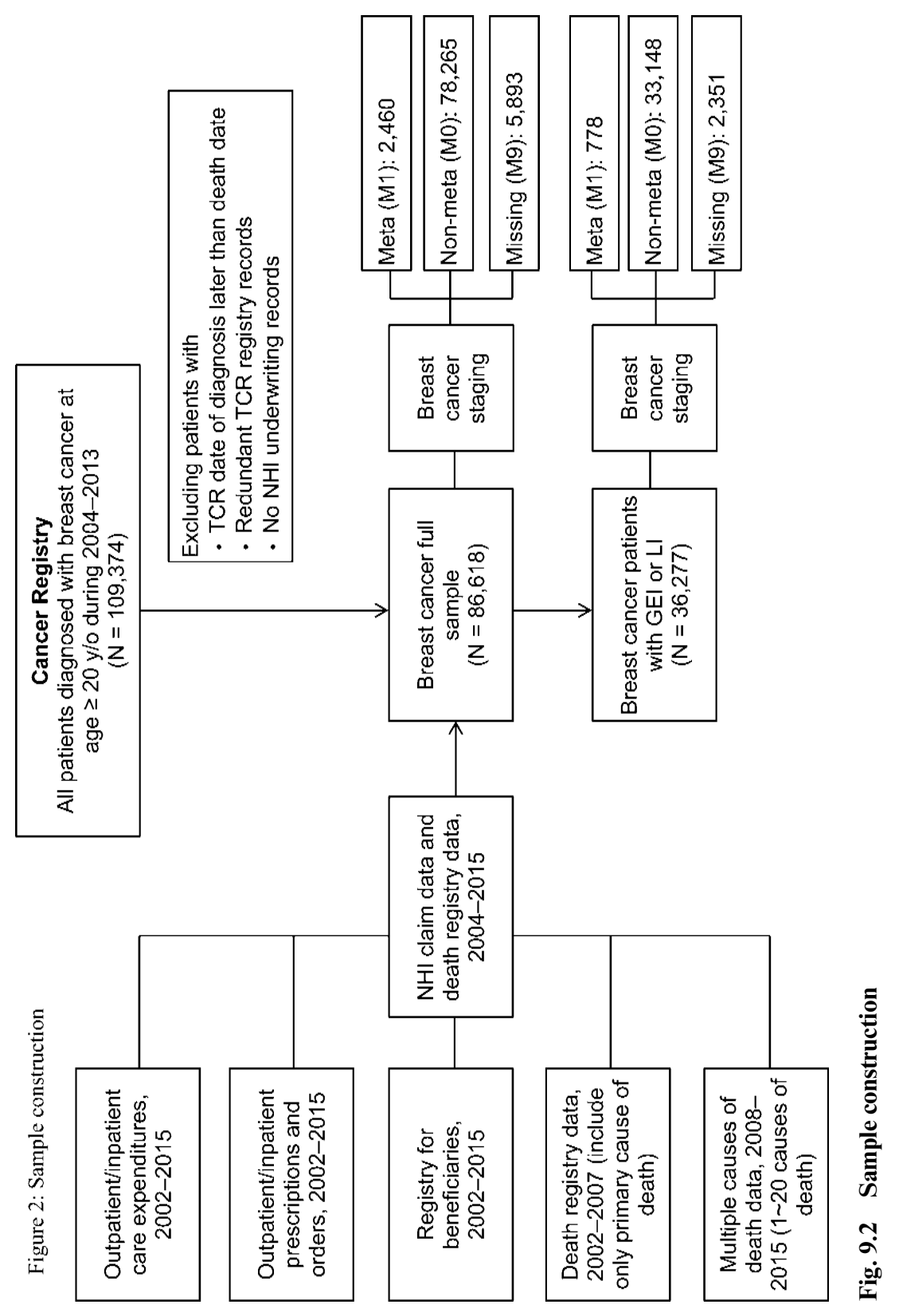


teachers in elementary, middle, and high schools and military service personnel were required to file tax returns. Contingent upon the income proxy we used, we have two analytical samples: for the full sample of patients, we use the district-specific median household income (from tax returns), and for the formal sector and government employees (hereafter, the GEI/LI sample), we use the reported monthly insured wage in the NHI Registry of Beneficiaries.

To examine potential disparities by geography, we also coded residency location for each patient. The NHI records the location of NHI enrollment, which is where the office of the employer is situated but not necessarily the residential location of the insured, particularly for those who work for large corporations. For example, big companies tend to set up headquarters in the capital city, Taipei, but many employees work at a factory or office outside the city (in a suburban or rural area) and reside near there. We hence adopted an algorithm to determine the residential location of a breast cancer patient based on her occupation (which corresponds with insured category) and where she utilizes primary care services (figure 9.3; Lin, Yang, and Wen 2011). Then the residency location is matched with district-specific median household income to obtain the economic status proxy for our study sample.

\subsubsection{Empirical Methods}

As one measure of income-related inequality in health care use (in this case, either diagnostic testing or target therapy), we computed a concentration index $(\mathrm{CI})$, with estimates for $\mathrm{CI}$ and its robust standard error obtained by running the following convenient regression (Kakwani, Wagstaff, and van Doorslaer 1997):

$$
\frac{2 \sigma_{R}^{2}}{\bar{y}} y_{i}=\alpha+\beta R_{i}+\varepsilon_{i}
$$

where $y$ is an indicator variable for whether the patient obtained the test or used target therapy $(0,1), \bar{y}$ is its mean, $R_{i}$ is the relative fractional rank of the $i$ th individual in the income distribution and $\sigma_{R}^{2}$ is the variance of $R_{i}$. The ordinary least squares (OLS) estimate of the slope coefficient $(\beta)$ is the estimate of CI.

When studying changes in utilization associated with the 2009 coverage of companion diagnostic testing with FISH (a specific test for breast cancer patients with suggestive, but not definitive, IHC testing results, such as $2+)$, it is most relevant to limit analysis to patients with metastatic disease at diagnosis (M1), the relevant group for comparing access before and after that policy change. However, given the limited sample size, concerns regarding missing data for M1, and the possibility of developing metastases after initial treatment, or case-by-case consideration of NHI coverage even for non-M1 cases, it is also instructive to compute $\mathrm{CI}_{\mathrm{TW}, t}$ at the national level for the entire sample for each year $(t=2002-2015)$ to see whether there is a trend across the years, and any jumps associated with the policy changes 


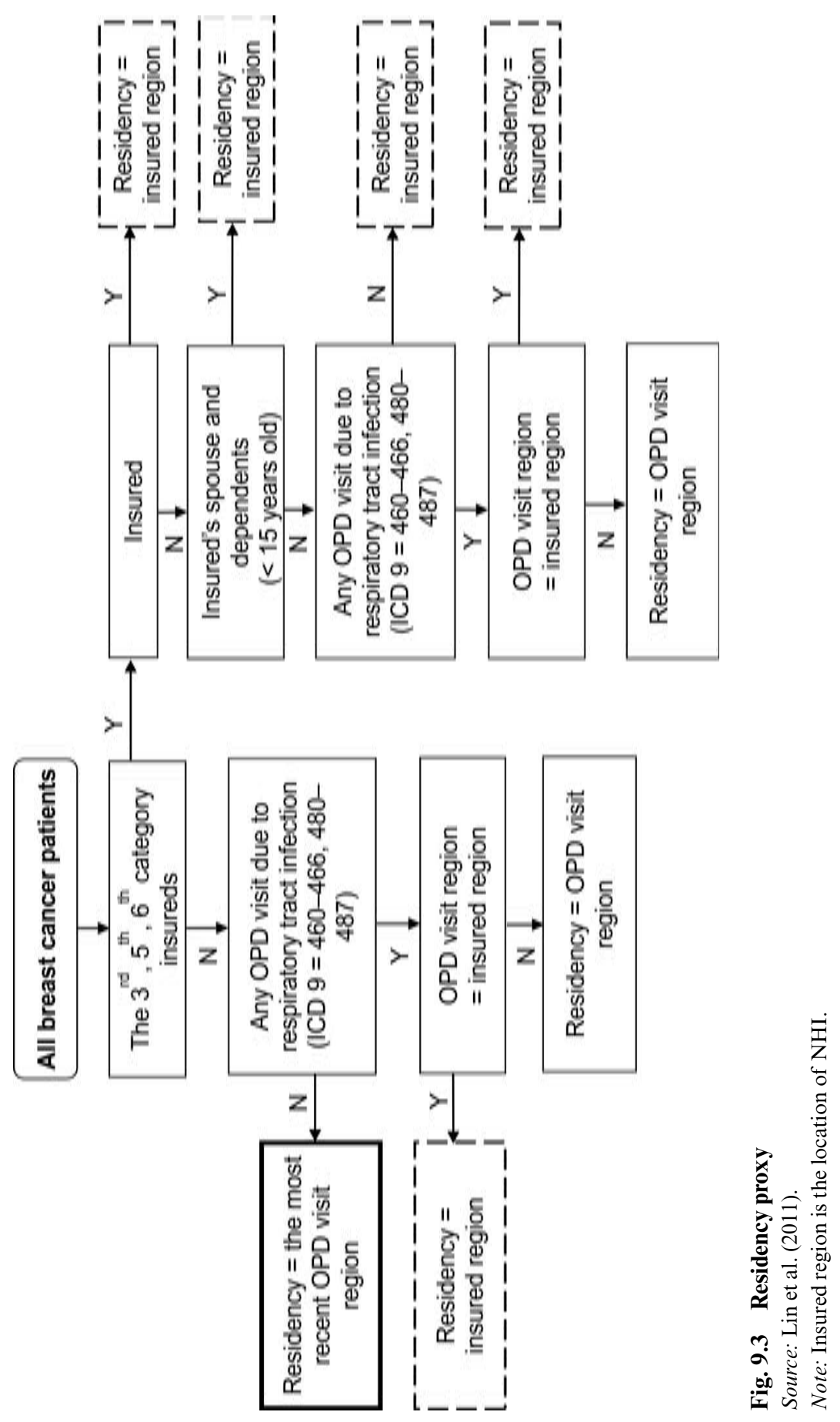


(i.e., FISH test coverage in 2009 and the extension of trastuzumab coverage to early stage cancer in 2010).

We also estimated logit regressions to test for disparities in diagnostic testing and target therapy utilization by income quintile, and its change after FISH testing was covered. The patient is categorized according to the year of diagnosis, recognizing that they continue treatment into subsequent years. We report the marginal effects from a logit regression to assess the association of utilization with income (Norton and Dowd 2017). Finally, as a preliminary examination of the correlates of patients' outcomes, we estimated Kaplan-Meier all-cause five-year survival curves by income quintile and by metastasis, adjusted for age and Charlson comorbidity index at diagnosis. Full examination of disparities in outcomes, accounting for censoring in our data, is left to future research.

\subsection{Results}

In our analytic sample of breast cancer patients in Taiwan, the year of diagnosis ranges from 2004 to 2013 . Roughly 62 percent (70 percent for the GEI/LI sample; see table 9.1) of breast cancer patients were diagnosed between the ages of forty to fifty-nine; the mean age at diagnosis was 53.46 years old (50.24 for the GEI/LI sample). Approximately 40 percent of the patients resided in the Taipei area (i.e., within the jurisdiction of the NHIA Taipei division office); within the sample of patients employed and insured under GEI or LI, there was a slightly higher concentration in the Taipei area (44.64 percent). The distribution of patients by tumor stage at diagnosis is 11.89 percent (13.52 percent for the GEI/LI sample) with tumor localized/in situ (Stage 0); the majority are diagnosed at an intermediate stage, and 2.84 percent (2.14 percent for GEI/LI sample) have metastatic breast cancer already at diagnosis $(M 1)$. The $\mathrm{M}$ staging variable is missing for 6.48-6.8 percent of the sample. As shown in table 9.1, average years survived is 5.57 years ( 5.69 years for GEI/LI sample), distributed from 0 to 11.99 years (survival is truncated at 12/2015). Among those in our sample, the year of death ranges from 2004 to 2015; 16.51 percent of the sample patients (11.94 percent for GEI/LI sample) died during the observation period (table 9.1). The Charlson Comorbidity Index score varies from 0 to 20; approximately 37.04 percent have a score of 2 and roughly 21.32 percent have a score of 8 (43.36 percent and 22.74 percent for GEI/LI sample, respectively; table 9.2).

Hypothesis 1 garners clear support in our data, suggesting that trastuzumab coverage may be pro-poor. In figure $9.4 \mathrm{~A}$, we show the proportion of patients who are late stage versus early stage by income quintile. For both samples and measures of income, there is a gradient with lower-income patients being more likely to be diagnosed with later stage cancer. For example, as hypothesized and consistent with earlier literature, the probability of metastasis at diagnosis is higher for those with lower income 


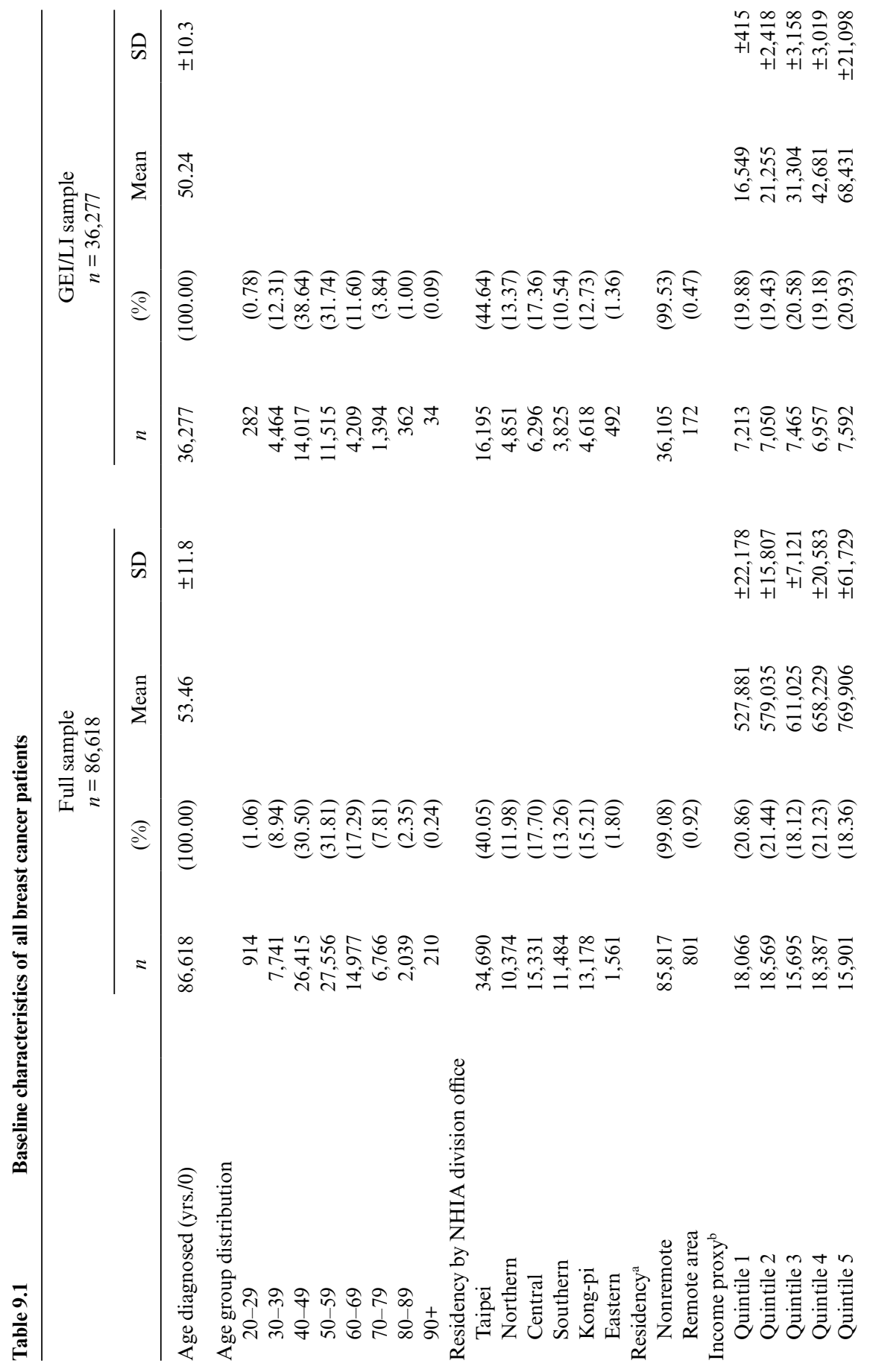




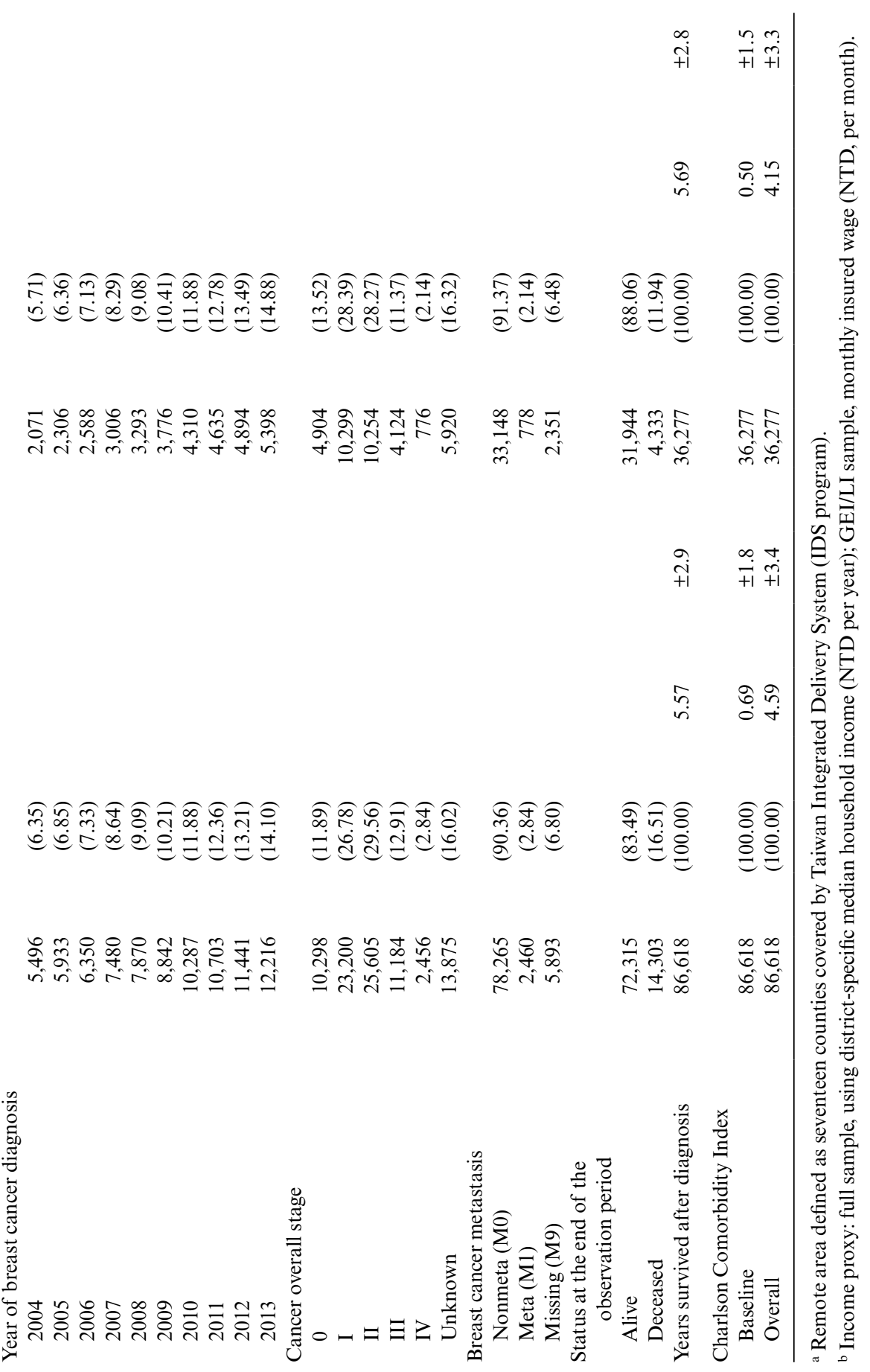


Table 9.2 Charlson Comorbidity Index (CCI) distribution

\begin{tabular}{|c|c|c|c|c|}
\hline & \multicolumn{2}{|c|}{$\begin{array}{c}\text { Full sample } \\
n=86,618\end{array}$} & \multicolumn{2}{|c|}{$\begin{array}{c}\text { GEI/LI sample } \\
n=36,277\end{array}$} \\
\hline & $n$ & $(\%)$ & $n$ & $(\%)$ \\
\hline \multicolumn{5}{|c|}{$\mathrm{CCI}$ at baseline $\mathrm{e}^{\mathrm{a}}$} \\
\hline 0 & 67,685 & (78.14) & 30,171 & (83.17) \\
\hline 1 & 4,108 & $(4.74)$ & 1,196 & (3.30) \\
\hline 2 & 7,918 & (9.14) & 3,088 & $(8.51)$ \\
\hline 3 & 2,245 & (2.59) & 592 & (1.63) \\
\hline 4 & 1,025 & (1.18) & 204 & $(0.56)$ \\
\hline 5 & 435 & $(0.50)$ & 90 & $(0.25)$ \\
\hline 6 & 288 & $(0.33)$ & 62 & $(0.17)$ \\
\hline 7 & 169 & $(0.20)$ & 30 & $(0.08)$ \\
\hline 8 & 1,828 & (2.11) & 662 & (1.82) \\
\hline 9 & 499 & $(0.58)$ & 107 & $(0.29)$ \\
\hline 10 & 239 & $(0.28)$ & 45 & $(0.12)$ \\
\hline 11 & 101 & $(0.12)$ & 16 & $(0.04)$ \\
\hline 12 & 38 & $(0.04)$ & 7 & $(0.02)$ \\
\hline $13+$ & 40 & $(0.04)$ & 7 & $(0.03)$ \\
\hline & & & - & - \\
\hline \multicolumn{5}{|c|}{$\mathrm{CCI}$ overall $^{\mathrm{b}}$} \\
\hline 0 & 6,766 & $(7.81)$ & 3,455 & $(9.52)$ \\
\hline 1 & 1,358 & (1.57) & 514 & (1.42) \\
\hline 2 & 32,080 & (37.04) & 15,731 & (43.36) \\
\hline 3 & 8,590 & $(9.92)$ & 2,885 & (7.95) \\
\hline 4 & 3,946 & $(4.56)$ & 1,157 & (3.19) \\
\hline 5 & 1,389 & (1.60) & 361 & (1.00) \\
\hline 6 & 774 & $(0.89)$ & 188 & $(0.52)$ \\
\hline 7 & 442 & $(0.51)$ & 90 & $(0.25)$ \\
\hline 8 & 18,485 & (21.34) & 8,251 & $(22.74)$ \\
\hline 9 & 6,354 & $(7.34)$ & 2,019 & $(5.57)$ \\
\hline 10 & 3,226 & $(3.72)$ & 851 & (2.35) \\
\hline 11 & 1,685 & (1.95) & 467 & (1.29) \\
\hline 12 & 730 & $(0.84)$ & 169 & $(0.47)$ \\
\hline 13 & 372 & $(0.43)$ & 55 & $(0.15)$ \\
\hline 14 & 218 & $(0.25)$ & 54 & $(0.15)$ \\
\hline $15+$ & 203 & $(0.24)$ & 30 & $(0.08)$ \\
\hline
\end{tabular}

${ }^{a} \mathrm{CCI}$ score calculated by comorbidity record before cancer diagnosed.

${ }^{\mathrm{b}} \mathrm{CCI}$ score calculated by comorbidity record in overall observation period.

(figure 9.4A and table 9.4, last column). In the full sample, 20.8 percent of women in the lowest income quintile are Stage III or IV, compared to 16.4 percent among those in the highest income quintile (figure 9.4B); similar patterns arise in the GEI/LI sample, with a 4.7 percentage points difference in late-stage (Stage III or IV) cancer between those in the lowest and highest income quintiles (figure 9.4C). Focusing only on those without metastasis, figure 9.5A shows that in the full sample, 17.6 percent of women in the low- 


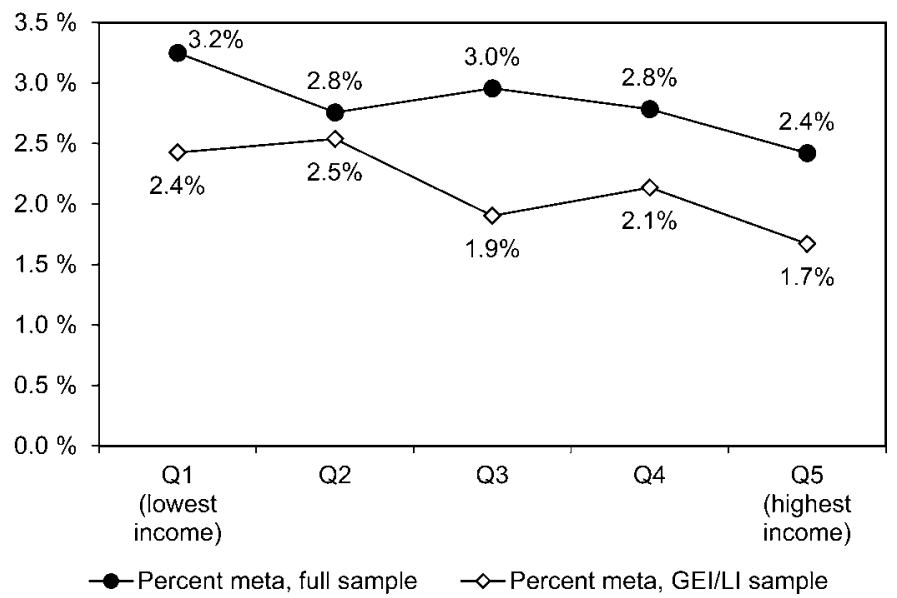

Fig. 9.4A Percentage of breast cancer patients with metastasis at diagnosis by income quintile

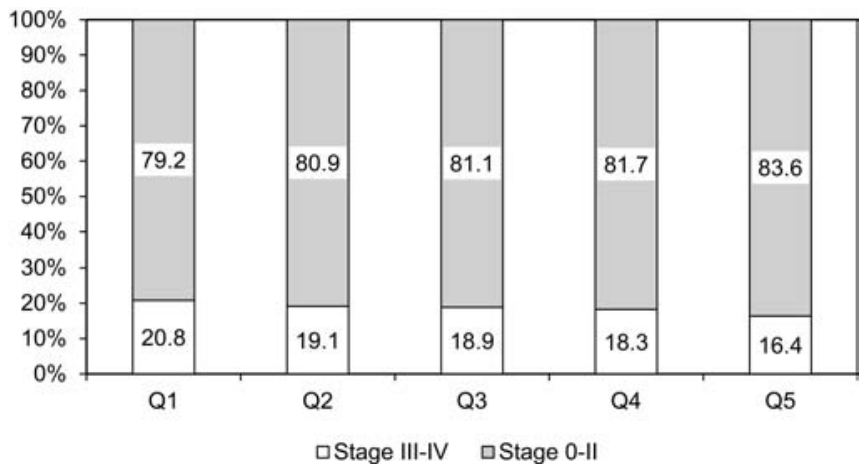

Fig. 9.4B Cancer stage by income quintile, full sample

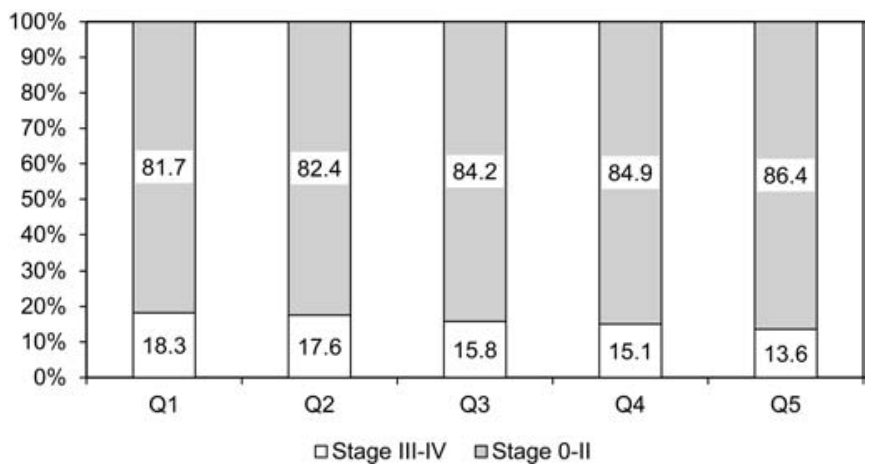

Fig. 9.4C Cancer stage by income quintile, GEI/LI sample 


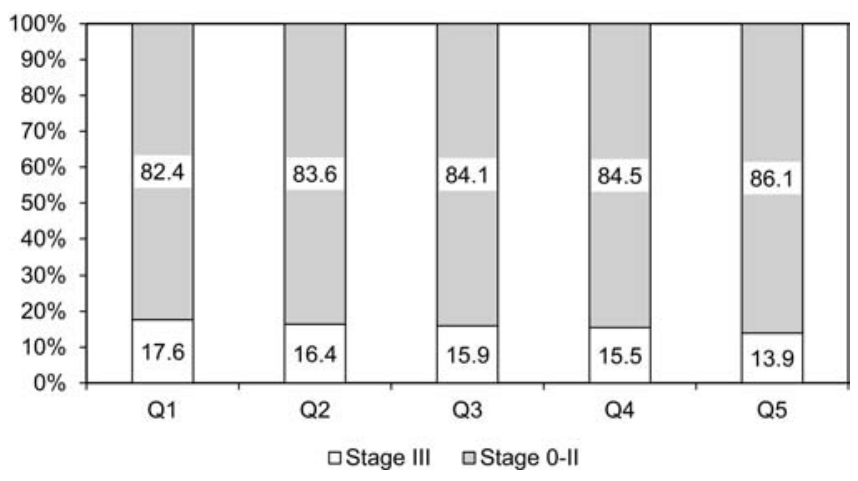

Fig. 9.5A Percentage of breast cancer patients diagnosed Stage 0-II and III by income quintiles in the full sample, 2004-2013

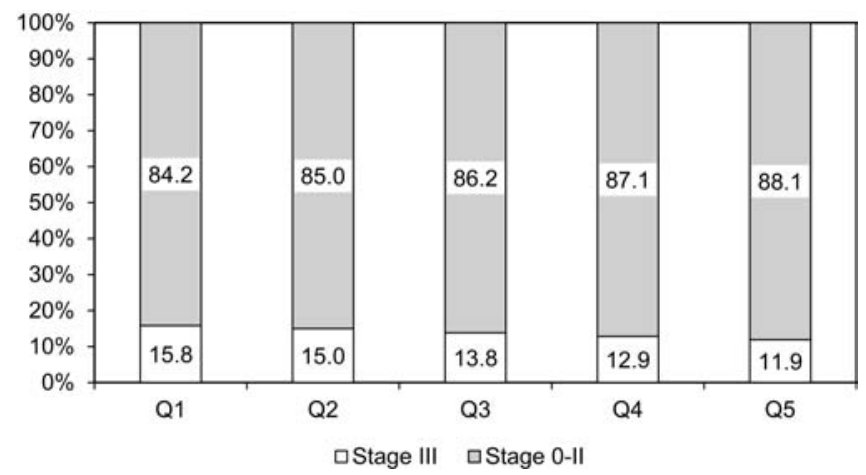

Fig. 9.5B Percentage of breast cancer patients diagnosed Stage 0-II and III by income quintiles in the GEI/LU sample, 2004-2013

est quintile of income are diagnosed as Stage III, compared to 13.9 percent of women in the highest income quintile. Among the GEI/LI sample, that difference is 15.8 percent compared to 11.9 percent (figure $9.5 \mathrm{~B}$ ).

We observe that about 79.35 percent ( 81.01 percent for GEI/LI sample) of sample patients had an IHC test (a general, low-cost test), 11.44 percent (11.88 percent for GEI/LI sample) had the FISH test, and roughly 10.82 percent ( 10.16 percent for GEI/LI sample) received target therapy with trastuzumab (tables 9.3A and 9.3B). Regarding testing, it is important to note that in up to 25 percent of cases, HER2 status may be discordant between the primary tumor and metastases, leading some to recommend (re-)testing of both primary tumor and the metastatic lesion at relapse (Loibl and Gianni 2017). Among patients receiving trastuzumab, most were treated at foundation-owned (private not-for-profit) hospitals, followed by public hospitals. Regarding the specialty of the primary provider, for both full and 


\begin{tabular}{|c|c|c|c|c|c|c|}
\hline & \multicolumn{6}{|c|}{$\begin{array}{c}\text { Full sample } \\
n=86,618\end{array}$} \\
\hline & \multicolumn{2}{|c|}{ Trastuzumab } & \multicolumn{2}{|c|}{ FISH test } & \multicolumn{2}{|c|}{ IHC test } \\
\hline & $n$ & $(\%)$ & $n$ & $(\%)$ & $n$ & $(\%)$ \\
\hline \multicolumn{7}{|c|}{ Patient was prescribed this test or therapy } \\
\hline Yes & 9,369 & (10.82) & 9,909 & (11.44) & 68,731 & (79.35) \\
\hline No & 77,249 & $(89.18)$ & 76,709 & $(88.56)$ & 17,887 & $(20.65)$ \\
\hline \multicolumn{7}{|c|}{ Ownership of hospital where prescribed } \\
\hline Public (government-owned) & 2,717 & $(29.00)$ & 3,875 & $(39.11)$ & 22,538 & $(32.79)$ \\
\hline $\begin{array}{l}\text { Foundation-owned (private } \\
\text { profit) }\end{array}$ & 6,377 & $(68.06)$ & 5,826 & $(58.80)$ & 44,161 & $(64.25)$ \\
\hline Physician-owned (private) & 275 & $(2.94)$ & 208 & $(2.10)$ & 2,032 & $(2.96)$ \\
\hline No prescribing & 77,249 & - & 76,709 & - & 17,887 & - \\
\hline \multicolumn{7}{|c|}{ Hospital location by NHIA division office } \\
\hline Taipei & 3,703 & $(39.52)$ & 4,056 & $(40.93)$ & 30,276 & $(44.05)$ \\
\hline Northern & 1,028 & $(10.97)$ & 1,088 & (10.98) & 7,820 & (11.38) \\
\hline Central & 1,831 & $(19.54)$ & 1,976 & (19.94) & 12,256 & $(17.83)$ \\
\hline Southern & 1,309 & $(13.97)$ & 1,073 & $(10.83)$ & 7,854 & (11.43) \\
\hline Kong-pi & 1,334 & $(14.24)$ & 1,626 & $(16.41)$ & 9,739 & (14.17) \\
\hline Eastern & 164 & $(1.75)$ & 90 & $(0.91)$ & 786 & (1.14) \\
\hline No prescribing & 77,249 & - & 76,709 & - & 17,887 & - \\
\hline \multicolumn{7}{|l|}{ Physician specialty } \\
\hline Surgery & 3,927 & $(41.91)$ & 6,470 & $(65.29)$ & 56,398 & $(82.06)$ \\
\hline Hematology \& oncology & 4,362 & $(46.56)$ & 2,081 & $(21.00)$ & 1,874 & $(2.73)$ \\
\hline Other & 1,080 & (11.53) & 1,358 & (13.70) & 10,459 & $(15.22)$ \\
\hline No prescribing & 77,249 & - & 76,709 & - & 17,887 & - \\
\hline \multicolumn{7}{|l|}{ Year prescribed } \\
\hline 2004 & 19 & $(0.20)$ & - & - & 3,498 & (5.09) \\
\hline 2005 & 103 & (1.10) & - & - & 4,175 & (6.07) \\
\hline 2006 & 280 & (2.99) & - & - & 4,662 & (6.78) \\
\hline 2007 & 366 & $(3.91)$ & - & - & 5,599 & (8.15) \\
\hline 2008 & 453 & $(4.84)$ & - & - & 5,933 & (8.63) \\
\hline 2009 & 547 & $(5.84)$ & 1,040 & $(10.50)$ & 6,894 & (10.03) \\
\hline 2010 & 1,744 & (18.61) & 1,863 & (18.80) & 7,931 & (11.54) \\
\hline 2011 & 1,620 & $(17.29)$ & 1,922 & (19.40) & 8,496 & (12.36) \\
\hline 2012 & 1,611 & $(17.20)$ & 2,027 & (20.46) & 9,353 & (13.61) \\
\hline 2013 & 1,616 & $(17.25)$ & 2,271 & (22.92) & 10,299 & (14.98) \\
\hline 2014 & 759 & $(8.10)$ & 525 & $(5.30)$ & 1,287 & (1.87) \\
\hline 2015 & 251 & (2.68) & 261 & $(2.63)$ & 604 & $(0.88)$ \\
\hline No prescribing & 77,249 & - & 76,709 & - & 17,887 & - \\
\hline
\end{tabular}




\begin{tabular}{|c|c|c|c|c|c|c|}
\hline & \multicolumn{6}{|c|}{$\begin{array}{c}\text { GEI/LI sample } \\
n=36,277\end{array}$} \\
\hline & \multicolumn{2}{|c|}{ Trastuzumab } & \multicolumn{2}{|c|}{ FISH } & \multicolumn{2}{|c|}{$\mathrm{IHC}$} \\
\hline & $n$ & $(\%)$ & $n$ & $(\%)$ & $n$ & $(\%)$ \\
\hline \multicolumn{7}{|c|}{ Patient was prescribed this test or therapy } \\
\hline Yes & 3,684 & $(10.16)$ & 4,308 & $(11.88)$ & 29,389 & $(81.01)$ \\
\hline No & 32,593 & $(89.84)$ & 31,969 & $(88.12)$ & 6,888 & (18.99) \\
\hline \multicolumn{7}{|c|}{ Ownership of hospital where prescribed } \\
\hline Public (government-owned) & 1,144 & $(31.05)$ & 1,826 & (42.39) & 10,263 & $(34.92)$ \\
\hline $\begin{array}{l}\text { Foundation-owned (private } 1 \\
\text { profit) }\end{array}$ & 2,433 & $(66.04)$ & 2,398 & $(55.66)$ & 18,317 & $(62.33)$ \\
\hline Physician-owned (private) & 107 & $(2.90)$ & 84 & $(1.95)$ & 809 & $(2.75)$ \\
\hline No prescribing & 32,593 & - & 31,969 & - & 6,888 & - \\
\hline \multicolumn{7}{|c|}{ Hospital location by NHIA division office } \\
\hline Taipei & 1,624 & (44.08) & 1,911 & $(44.36)$ & 14,311 & $(48.70)$ \\
\hline Northern & 469 & $(12.73)$ & 491 & $(11.40)$ & 3,586 & $(12.20)$ \\
\hline Central & 710 & (19.27) & 873 & (20.26) & 4,971 & $(16.91)$ \\
\hline Southern & 389 & $(10.56)$ & 369 & $(8.57)$ & 2,765 & $(9.41)$ \\
\hline Kong-pi & 442 & $(12.00)$ & 632 & $(14.67)$ & 3,528 & $(12.00)$ \\
\hline Eastern & 50 & $(1.36)$ & 32 & $(0.74)$ & 228 & $(0.78)$ \\
\hline No prescribing & 32,593 & - & 31,969 & - & 6,888 & - \\
\hline \multicolumn{7}{|l|}{ Physician specialty } \\
\hline Surgery & 1,576 & $(42.78)$ & 2,822 & $(65.51)$ & 24,361 & (82.89) \\
\hline Hematology \& oncology & 1,693 & $(45.96)$ & 888 & $(20.61)$ & 693 & $(2.36)$ \\
\hline Other & 415 & (11.26) & 598 & $(13.88)$ & 4,335 & $(14.75)$ \\
\hline No prescribing & 32,593 & - & 31,969 & - & 6,888 & - \\
\hline \multicolumn{7}{|l|}{ Year prescribed } \\
\hline 2004 & 6 & $(0.16)$ & - & - & 1,379 & $(4.69)$ \\
\hline 2005 & 37 & $(1.00)$ & - & - & 1,675 & $(5.70)$ \\
\hline 2006 & 89 & $(2.42)$ & - & - & 1,932 & (6.57) \\
\hline 2007 & 114 & $(3.09)$ & - & - & 2,255 & (7.67) \\
\hline 2008 & 147 & $(3.99)$ & - & - & 2,534 & (8.62) \\
\hline 2009 & 191 & $(5.18)$ & 459 & (10.65) & 3,029 & (10.31) \\
\hline 2010 & 712 & (19.33) & 781 & (18.13) & 3,384 & (11.51) \\
\hline 2011 & 671 & (18.21) & 821 & (19.06) & 3,746 & (12.75) \\
\hline 2012 & 637 & (17.29) & 889 & (20.64) & 4,060 & (13.81) \\
\hline 2013 & 664 & $(18.02)$ & 1,014 & (23.54) & 4,600 & $(15.65)$ \\
\hline 2014 & 314 & $(8.52)$ & 232 & $(5.39)$ & 566 & (1.93) \\
\hline 2015 & 102 & $(2.77)$ & 112 & $(2.60)$ & 229 & $(0.78)$ \\
\hline No prescribing & 32,593 & - & 31,969 & - & 6,888 & - \\
\hline
\end{tabular}


GEI/LI samples, roughly 82 percent/65 percent ( 83 percent/65.5 percent for the GEI/LI sample) of the sample patients had IHC/FISH testing managed by a surgeon, compared to 3 percent $/ 21$ percent ( 2 percent $/ 20.6$ percent for the GEI/LI sample) by an oncologist. However, approximately 47 percent of the sample patients had trastuzumab prescribed by an oncologist, and 42 percent by a surgeon ( 46 percent and 42.8 percent, respectively, for the GEI/ LI sample; tables 9.3A and 9.3B).

We tested our primary hypotheses by estimating a concentration index for different samples of patients and different years. In addition to the full sample and GEI/LI sample of all-stage cancer patients, we also examined the income-related inequality in the use of IHC and FISH tests and trastuzumab for the M1 metastatic sample (who would not be affected by the extension of indication to M0 patients since 2010). As shown by the CIs in table 9.4, the use of IHC companion diagnostic testing shows a pro-rich distribution and the utilization of FISH companion diagnostic testing tends to be proportionally distributed. A pro-poor inequality in the use of trastuzumab is observed, although the magnitude appears to be modest. Overall, the significance and magnitude of the inequality are more obvious in the full sample than the GEI/LI sample, that is, those for whom we have the more accurate income proxy based on monthly wage. Confirming the summary statistics shown in figure 9.4A, metastasis at initial diagnosis (last column in table 9.4) exhibits a marginally pro-poor distribution of most advanced (i.e., already metastatic) breast cancer.

Further, we aggregate the observations into pre- and postpolicy periods to examine patterns of use before and after the expansion of coverage by M status (table 9.5). We define the prepolicy period (labeled "preperioda") as 2002-2008 to represent the period before FISH was covered by NHIA, except in the case of the nonmetastatic sample, where the preperiod is 2002 2009 (before the extension of indication to cover M0 patients). Accordingly, the postperiod is 2009-2015 for the "all" sample and M1 sample (after FISH is covered by NHI), but 2010-2015 for the M0 sample (after the extension of indication to cover M0 patients). Aside from the definition above (preperiod $^{a}$ ), to try to disentangle the effect of the coverage expansions from the natural course of the disease (e.g., M0 patients developing metastases in the pre-2010 period and gaining access to trastuzumab), we also deleted patients diagnosed in the prepolicy years but who received testing or trastuzumab in the postpolicy period (labeled preperiod ${ }^{\mathrm{b}}$ ).

All the CIs that are statistically significant both for the full sample and for the GEI/LI sample in any of the periods are negative, showing the distribution of the use of trastuzumab is pro-poor (table 9.5). The overall CI for the full sample is -0.0124 , a modest magnitude. The magnitude of the postperiod CI is slightly larger than the preperiod CI, suggesting that the expanded coverage strengthened access for the poor. Comparing the two definitions of the preperiod, we see that preperiod ${ }^{\mathrm{a}}$ estimated $\mathrm{CI}$ is more 
Table 9.4

Income-related inequality in use of trastuzumab, IHC, FISH gene-testing and breast cancer metastasis, as measured by concentration index by year of diagnosis

\begin{tabular}{|c|c|c|c|c|c|c|}
\hline \multirow[b]{2}{*}{$\begin{array}{l}\text { Year of } \\
\text { diagnosis }\end{array}$} & \multicolumn{6}{|c|}{ Full sample } \\
\hline & $n$ & $\begin{array}{c}\mathrm{IHC} \\
\mathrm{CI}\end{array}$ & $\begin{array}{c}\text { FISH } \\
\text { CI }\end{array}$ & $\begin{array}{c}\text { Trastuzumab } \\
\text { CI }\end{array}$ & $n$ & $\begin{array}{c}\text { Breast cancer } \\
\text { metastasis } \\
\text { CI }\end{array}$ \\
\hline Overall & 86,618 & $0.0247 * * *$ & -0.0024 & $-0.0133 * * *$ & 80,725 & $-0.0009 * * *$ \\
\hline 2004 & 5,496 & 0.0052 & -0.0002 & 0.0039 & 5,247 & -0.0003 \\
\hline 2005 & 5,933 & $0.0580 * * *$ & -0.0007 & $-0.0174 * * *$ & 5,639 & -0.0025 \\
\hline 2006 & 6,350 & $0.0253^{*}$ & -0.0011 & $-0.0113 * *$ & 6,023 & -0.0025 \\
\hline 2007 & 7,480 & $0.0316^{* *}$ & $-0.0034 * * *$ & $-0.0128 * * *$ & 6,924 & $-0.0017 * *$ \\
\hline 2008 & 7,870 & $0.0224^{*}$ & -0.0015 & $-0.0077 * *$ & 7,259 & -0.0004 \\
\hline 2009 & 8,842 & $0.0219 *$ & -0.0021 & $-0.0185^{* * *}$ & 8,165 & $-0.0017 * *$ \\
\hline 2010 & 10,287 & $0.0284 * *$ & -0.0074 & $-0.0116^{*}$ & 9,651 & -0.0007 \\
\hline 2011 & 10,703 & $0.0179 *$ & -0.0097 & $-0.0180 * *$ & 9,919 & 0.0000 \\
\hline 2012 & 11,441 & $0.0209 *$ & 0.0131 & $-0.0107^{*}$ & 10,582 & -0.0003 \\
\hline \multirow[t]{2}{*}{2013} & 12,216 & $0.0268 * * *$ & $0.0193^{* *}$ & $-0.0155^{* * *}$ & 11,316 & $-0.0017^{* *}$ \\
\hline & \multicolumn{6}{|c|}{ GEI/LI sample } \\
\hline Overall & 36,277 & 0.0063 & $-0.0083^{* * *}$ & $-0.0126^{* * *}$ & 33,926 & $-0.0007 * *$ \\
\hline 2004 & 2,071 & 0.0079 & -0.0028 & $-0.0089 *$ & 1,994 & $-0.0033^{*}$ \\
\hline 2005 & 2,306 & 0.0287 & -0.0016 & -0.0087 & 2,196 & -0.0012 \\
\hline 2006 & 2,588 & 0.0043 & 0.0006 & -0.0065 & 2,470 & $-0.0047^{*}$ \\
\hline 2007 & 3,006 & 0.0047 & -0.0019 & -0.0052 & 2,806 & $-0.0010^{*}$ \\
\hline 2008 & 3,293 & 0.0035 & $-0.0052 *$ & -0.0063 & 3,070 & -0.0002 \\
\hline 2009 & 3,776 & $0.0429 * * *$ & -0.0047 & -0.0134 & 3,517 & -0.0003 \\
\hline 2010 & 4,310 & 0.0150 & 0.0006 & -0.0081 & 4,049 & $-0.0014^{*}$ \\
\hline 2011 & 4,635 & -0.0096 & -0.0148 & $-0.0192 *$ & 4,303 & 0.0004 \\
\hline 2012 & 4,894 & 0.0090 & -0.0143 & $-0.0186^{* *}$ & 4,542 & -0.0003 \\
\hline 2013 & 5,398 & -0.0173 & -0.0053 & $-0.0155^{* *}$ & 4,979 & -0.0002 \\
\hline
\end{tabular}

Notes: Breast cancer metastasis $=$ the analytical sample excluding patients with $\mathrm{M}$ staging missing value; income proxy = full sample, using district-specific median household income (NTD per year); GEI/LI sample, monthly insured wage (NTD, per month); CI = Concentration Index.

$* * *$ Significant at the 0.1 percent level.

**Significant at the 1 percent level.

* Significant at the 5 percent level.

pro-poor than that of preperiod $^{\mathrm{b}}$, which excludes those who gained delayed access to trastuzumab in the postperiod. This difference implies that more poor patients gained delayed access than rich patients did, both in the full sample and the GEI/LI sample. The pro-poor tendency in the all-stage cancer sample (M0 + M1) mainly comes from the much larger sample of M0 patients, where the CI for preperiod ${ }^{\text {a }}$ (which includes those with delayed access) is significantly more pro-poor than the CI for preperiod ${ }^{\mathrm{b}}$. For those with nonmissing codes for $\mathrm{M}$ and metastatic cancer at diagnosis (i.e., the M1 
Income-related inequality in use of trastuzumab for patients diagnosed before and after coverage expansion, as measured by concentration index

\begin{tabular}{|c|c|c|c|c|}
\hline \multirow{3}{*}{$\begin{array}{l}\text { M0 + M1 patients } \\
\text { Time of diagnosis }\end{array}$} & \multicolumn{2}{|c|}{ Full sample } & \multicolumn{2}{|c|}{ GEI/LI sample } \\
\hline & \multicolumn{2}{|c|}{$n=78,265$} & \multicolumn{2}{|c|}{$n=33,926$} \\
\hline & $n$ & $\mathrm{CI}$ & $n$ & CI \\
\hline All & 80,725 & $-0.0124 * * *$ & 33,926 & $-0.0112 * * *$ \\
\hline Preperiod $^{\mathrm{a}}$ & 39,046 & $-0.0107^{* * *}$ & 15,976 & $-0.0076^{* * *}$ \\
\hline Preperiod $^{\mathrm{b}}$ & 37,241 & $-0.0028 * * *$ & 15,249 & $-0.0027^{* * *}$ \\
\hline Postperiod & 41,679 & $-0.0119 * * *$ & 17,950 & $-0.0136^{* * *}$ \\
\hline \multirow{2}{*}{$\begin{array}{l}\text { M0 patients } \\
\text { Time of diagnosis }\end{array}$} & \multicolumn{2}{|c|}{$n=78,265$} & \multicolumn{2}{|c|}{$n=33,148$} \\
\hline & $n$ & $\mathrm{CI}$ & $n$ & $\mathrm{CI}$ \\
\hline All & 78,265 & $-0.0106^{* * *}$ & 33,148 & $-0.0107 * * *$ \\
\hline Preperiod $^{\mathrm{a}}$ & 37,925 & $-0.0094 * * *$ & 15,645 & $-0.0066^{* * *}$ \\
\hline Preperiod $^{b}$ & 36,167 & $-0.0019^{* * *}$ & 14,937 & $-0.0021 * * *$ \\
\hline Postperiod & 40,340 & $-0.0098 * * *$ & 17,503 & $-0.0138 * * *$ \\
\hline \multirow{2}{*}{$\begin{array}{l}\text { M1 patients } \\
\text { Time of diagnosis }\end{array}$} & \multicolumn{2}{|c|}{$n=2,460$} & \multicolumn{2}{|c|}{$n=778$} \\
\hline & $n$ & $\mathrm{CI}$ & $n$ & $\mathrm{CI}$ \\
\hline All & 2,460 & $-0.0616^{*}$ & 778 & 0.0605 \\
\hline Preperiod $^{\mathrm{a}}$ & 1,121 & -0.0508 & 331 & 0.0230 \\
\hline Preperiod $^{b}$ & 1,074 & $-0.0677^{*}$ & 312 & 0.0052 \\
\hline Postperiod & 1,339 & -0.0585 & 447 & 0.0899 \\
\hline
\end{tabular}

Notes: $\mathrm{CI}=$ Concentration Index; income proxy $=$ full sample, using district-specific median household income (NTD per year); GEI/LI sample, monthly insured wage (NTD, per month); preperiod $^{\mathrm{a}}=$ year of diagnosis $\leqq 2009$ for M0 $\leqq 2008$ for M1 preperiod ${ }^{\mathrm{b}}=$ same definition as preperiod $^{\mathrm{a}}$, but excluding patients who received trastuzumab after 2010 for M0, and 2009 for M1.

*** Significant at the 0.1 percent level.

$* *$ Significant at the 1 percent level.

*Significant at the 5 percent level.

sample), the CI are only marginally statistically significant (albeit of larger magnitude than for M0), although several estimates are indistinguishable from zero.

As a robustness check to the $\mathrm{CI}$ analyses, we also examined the likelihood of receiving target therapy by income quintile, controlling for age at diagnosis and comorbidities. These descriptive regressions (tables 9.6 and 9.7) confirm that the policy of better insurance coverage (for the FISH diagnostic test as well as for earlier stages of breast cancer) is associated with a statistically significant increase in trastuzumab use. The postpolicy-estimated coefficient is significant for almost all samples. The highest-income nonmetastatic patients were less likely to receive trastuzumab in the preperiod 


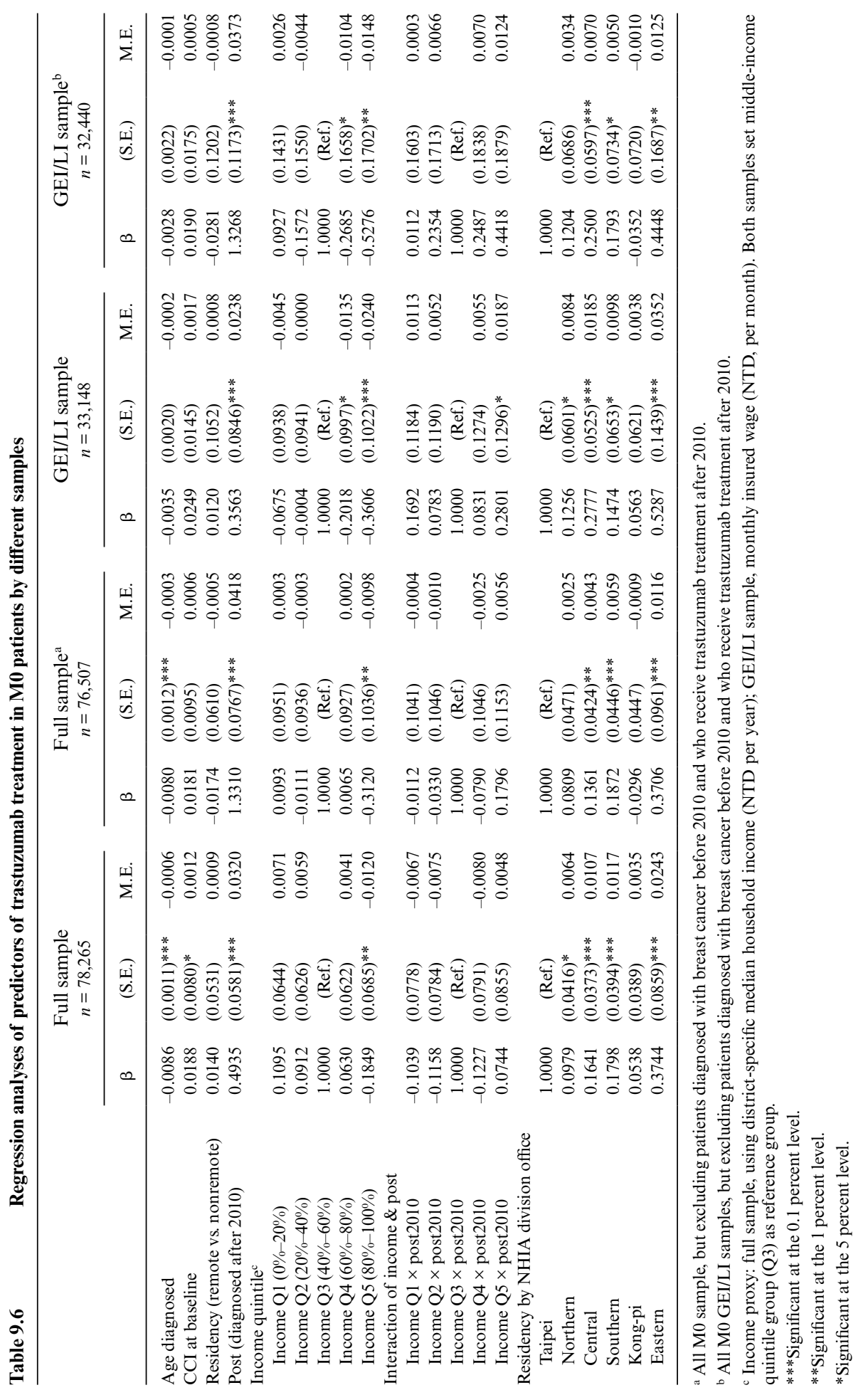




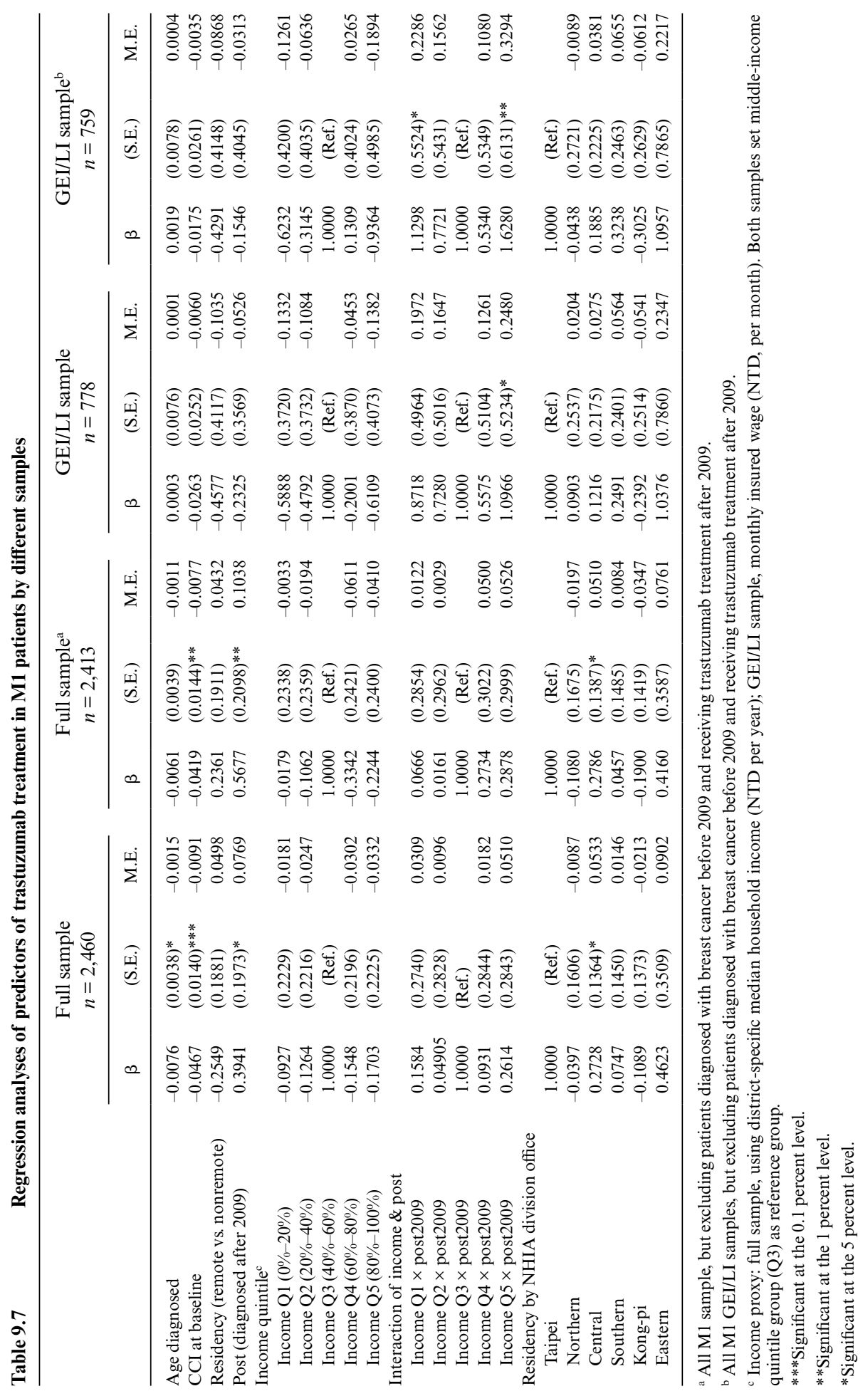


(table 9.6), consistent with their greater likelihood of having the earliest stage of cancer. The interaction of post and income is not significant, although the region of residence indicator variables (which are also correlated with SES) are jointly significant. Among metastastic cancer cases, trastuzumab treatment is unrelated to regional median income, although there is some evidence that those with both the lowest and highest insured wage quintiles received more NHI-covered target therapy in the postperiod (see table 9.7). We find slightly stronger results when excluding patients who were diagnosed prior to the policy change, but who received the FISH test after the policy change. There is only weak support for Hypothesis 3 , that the proportion of lower-income patients receiving target therapy increases after the FISH test is covered by insurance. This might be expected: only a small proportion of patients will fall in the intermediate zone where an IHC is ambiguous but a FISH test would be definitive, and it is difficult to tease out exactly which patients benefited since (a) we do not observe test results and (b) the FISH test coverage happened so close to the 2010 policy change affecting a much larger proportion of patients.

Thus, overall we find that among women diagnosed with $\mathrm{M} 0$ breast cancer before 2010, trastuzumab use was higher among low-income than highincome women (the $\mathrm{CI}$ is negative, and the regression shows patients in the highest-income quintile were less likely to receive trastuzumab). This is consistent with poorer women being diagnosed at later stages and more likely to develop metastases (e.g., in our data seventy patients diagnosed M0 in 2005 were prescribed trastuzumab in 2007). When examining the differences between M0 patients who receive trastuzumab before and after 2010, we find the pre-2010 users were more likely Stage III and far more likely to die during our study period, as would be the case for those women who developed metastatic cancer. Of course, the differential could also be because highincome women are more likely self-paying for target treatment not covered by NHI (which we cannot directly observe in this data), or other reasons.

An examination of survival differences - accounting for censoring, differential "missingness" of staging and other factors - is left for future research. However, it is worth noting that, examining figures 9.6A and 9.6B from previous analyses performed, we do observe the highest-income patients enjoying longer survival in both the full sample (figure 9.6A) and the GEI/ LI sample (figure 6b; Lu, Eggleston, and Chang 2018). Unsurprisingly, the primary differentiator for survival is cancer staging, with metastatic cancer associated with significantly lower survival (figure 6c; Lu, Eggleston, and Chang 2018). The survival curve for those with missing data on metastasis lies in between the curves for metastatic and nonmetastatic cases; this pattern suggests that some of those with missing meta status did indeed have metastatic breast cancer. A corollary of our simple model and hypotheses would be that, conditional on stage of cancer at diagnosis, both before and after the coverage expansions, the proportion receiving combined target 


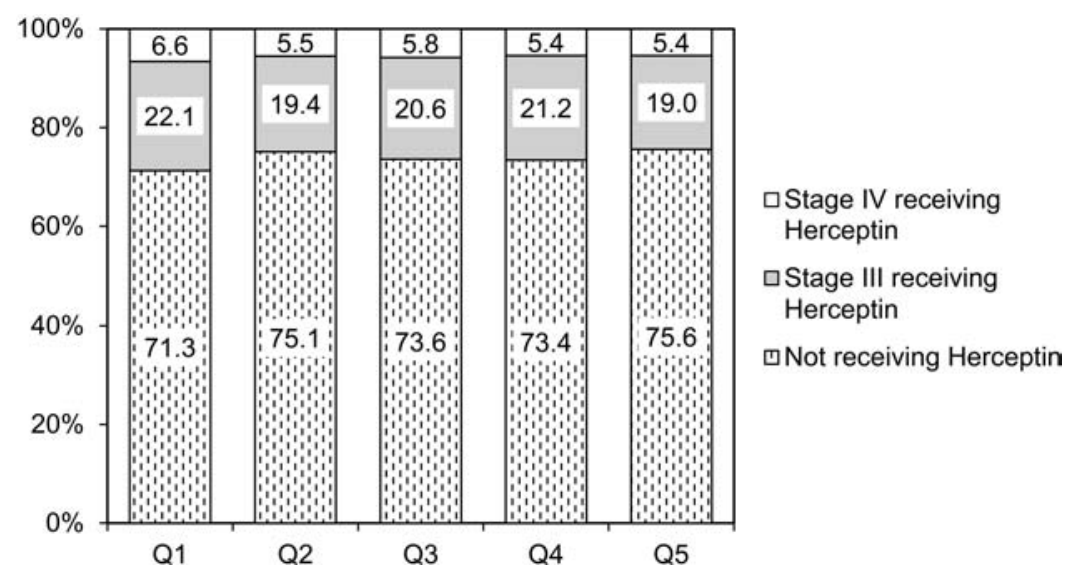

Fig. 9.6A Probability of receiving trastuzumab by income quintiles among stage III \& IV patients of the full sample diagnosed between 2004 and 2013

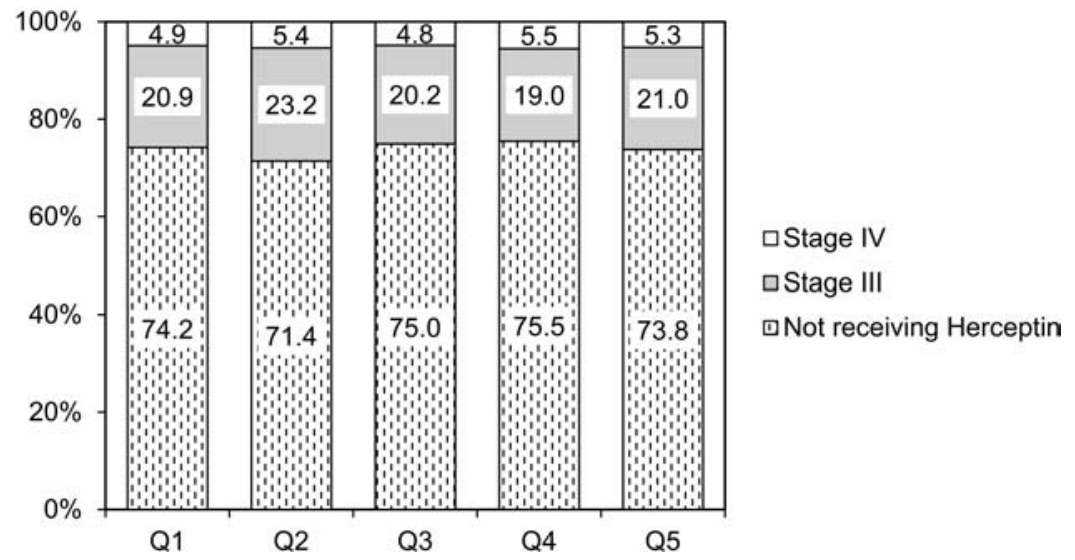

Fig. 9.6B Probability of receiving trastuzumab by income quintiles among stage III \& IV patients of the GEI/LI sample diagnosed between 2004 and 2013

therapy by paying out of pocket for pertuzumab increases with income, resulting in longer survival for higher-income patients. Both earlier stage at diagnosis and access to self-paid combination therapies may contribute to the observed longer survival of those with higher income, evident at three to five years for the GEI/LI sample (figure 6b of Lu, Eggleston, and Chang 2018). That access to combination therapy probably plays a role in this survival differential is suggested by the fact that, conditional on stage at diagnosis, the likelihood of receiving trastuzumab differs only slightly across income quintiles (figures 9.6A and 9.6B of this chapter). 


\subsection{Discussion and Conclusion}

We examine the case of breast cancer treatment in Taiwan to exemplify the potential of PPM to be pro-poor, with coverage extensions further expanding access to PPM treatment and reducing disparities. Although not all health systems can afford such comprehensive coverage as Taiwan's NHI provides for breast cancer patients, and we have not assessed the overall welfare impact, including the opportunity costs of the associated expenditure increases, Taiwan's experience illustrates that PPM coverage can disproportionately benefit the poor, even when introduced without full coverage of the companion diagnostic tests.

Specifically, we hypothesized that the overall association between patient income and receipt of trastuzumab therapy may be positive or negative, and the greater the propensity for low SES patients to present with later stage disease, the more likely that target therapy utilization is pro-poor (i.e., with a negative concentration index). The data show that lower-income patients are indeed more likely to present with later stage disease (more advanced stages of breast cancer) in Taiwan. For the most advanced stage, the distribution of metastatic cases is marginally pro-poor for both proxies for income, although the magnitude is modest.

Perhaps more important, we find that the inequality in use of trastuzumab treatment, as measured by the CIs among patients with all stages of cancer, are pro-poor, statistically significant, and are larger in magnitude in the postpolicy period than the prepolicy period (table 9.5). These patterns are consistent between the full sample and the sample of employed formal sector workers with a more accurate individual-level income proxy (GEI/ LI sample). Hence, it seems that the fact that lower SES patients are more likely to be diagnosed with later stages of cancer outweighs the presumed access advantage possessed by the rich, rendering NHI coverage of target therapy pro-poor even before coverage of the diagnostic test. Moreover, those who gained "delayed access" by the expansion of NHI coverageincluding the FISH diagnostic test and of target therapy for earlier stages of breast cancer - were disproportionately poor, strengthening the pro-poor distribution of target treatment.

The lack of a significant impact of NHI coverage of FISH tests for propoor access suggests that the costs of testing did not significantly deter lowincome patients from treatment, either because the testing costs are small relative to those of therapy or because the testing costs were covered by others in ways we cannot observe in our data prior to NHI coverage, such as coverage by pharmaceutical companies or provider research and access funds.

When looking at genetic testing in our data, we found that the use of IHC, which is a low-cost genetic test that is not specific to breast cancer, 
shows a pro-rich inequality in use, whereas no income-related inequality is found among users of the FISH test. This may imply that the well-off have better awareness and access to general genetic testing, although access to the more specialized and expensive test (which we only observe after it is covered by NHI) is not disproportionately used by those with higher income. In ongoing research, we are studying the prevalence and possible impact of pharmaceutical companies' coverage of companion diagnostic testing in Taiwan and other parts of East Asia.

To assist with putting these results in policy perspective, we develop a back-of-the-envelope estimate of the total life-years saved by the NHI expanded coverage policies for both metastatic and early stage breast cancer patients. We estimate that for women diagnosed with breast cancer in the four-year period 2010-2013, NHI trastuzumab coverage policy led to an additional 6,600 to 33,200 life-years, depending on the range of the estimates of life-years saved by trastuzumab treatment for early stage (from one to five years) and metastatic breast cancer ( 0.25 to 1.2 years), with a preferred estimate of about 20,000 life-years. ${ }^{6}$

The data does not include those who self-pay for target therapy. Therefore our estimate of disparities cannot account for the number of (presumably higher-income) individuals paying entirely out of pocket for testing and target therapy, especially in the early years before the treatment became standard of care for HER2-positive breast cancer. In future research, we will examine the data available in the Taiwan cancer registry starting in 2011 that records whether a patient received target therapy (even if such therapy was not covered by NHI). If that data is coded with reasonable completeness, it will enable us to observe the distribution of target therapy for those who have breast cancer but who are not receiving treatment covered by NHI. We also will examine patient records for a large provider that includes data on all treatments received, including both NHI-covered services (e.g., trastuzumab) and self-paid services (e.g., pertuzumab).

Future research will also explore potential interactions with supply-side incentives based on fee-for-service or bundled payment (Wang, Chen, and Wu 2017), specialty of provider, level of accreditation, and ownership of the clinic or hospital. Finally, an extension will contrast the case of breast cancer with that of colorectal cancer, where pharmaceutical firms pay for the companion diagnostic tests in exchange for NHI coverage of their target therapy.

6. For patients with metastastic breast cancer, we estimate trastuzumab extends survival by three months, but with a range of up to fourteen months for those who also self-pay for pertuzumab (Danese et al. 2015). Estimates of life-years saved for patients with early stage breast cancer come from the literature (see, e.g., Romond et al. 2005; Slamon et al. 2011; Perez et al. 2014), with the preferred estimate from Garrison et al. (2007), who projected life expectancy was three years longer for patients who received trastuzumab (19.4 years vs. 16.4 years). 


\section{References}

Appold, Karen. 2017. "Health Plans Explain Genetic Testing Coverage Decisions." Managed Healthcare Executive, Aug. 24. Accessed Mar. 6, 2018. http:// managedhealthcareexecutive.modernmedicine.com/managed-healthcare-executive /news/health-plans-explain-genetic-testing-coverage-decisions.

Asano, Hokuto. 2017. "Personalized and Precision Medicine in Japan." Working Paper no. 43, Stanford Asia Health Policy Program.

Chan, C., S. C. Ho, S. G. Chan., Y. B. Yip, F. C. Wong, and F. Cheng. 2002. "Factors Affecting Uptake of Cervical and Breast Cancer Screening among Perimenopausal Women in Hong Kong." Hong Kong Medical Journal 8 (5): 334-41.

Chang, Chun-Ming, Yu-Chieh Su, Ning-Sheng Lai, Kuang-Yung Huang, Sou-Hsin Chien, Yu-Han Chang, Wei-Cheng Lian, Ta-Wen Hsu, and Ching-Chih Lee. 2012. "The Combined Effect of Individual and Neighborhood Socioeconomic Status on Cancer Survival Rates." PLoS ONE 7 (8): e44325.

Chiang, Chun-Ju, Yong-Chen Chen, Chien-Jen Chen, San-Lin You, and Mei-Shu Lai. Taiwan Cancer Registry Task Force. 2010. "Cancer Trends in Taiwan." Japanese Journal of Clinical Oncology 40 (10): 897-904.

Chiang, Chun-Ju, San-Lin You, Chien-Jen Chen, Ya-Wen Yang, Wei-Cheng Lo, and Mei-Shu Lai. 2015. "Quality Assessment and Improvement of Nationwide Cancer Registration System in Taiwan: A Review." Japanese Journal of Clinical Oncology 45 (3): 291-96.

Daly, Bobby, and Olufunmilaya I. Olopade. 2015. "A Perfect Storm: How Tumor Biology, Genomics, and Health Care Delivery Patterns Collide to Create a Racial Survival Disparity in Breast Cancer and Proposed Interventions for Change." $C A$ : A Cancer Journal for Clinicians 65 (3): 221-38.

Danese, Mark D., Anthony Masaquel, Eduardo Santos, Melissa Brammer, Abraham Lee, and Deepa Lalla. 2015. "Estimated Life-Years Saved in Women with HER2-Positive Metastatic Breast Cancer Receiving First-Line Trastuzumab and Pertuzumab in the United States." Value in Health 18 (6): 876-83.

DeSantis, Carol D., Stacey A. Fedewa, Ann Goding Sauer, Joan L. Kramer, Robert A. Smith, and Ahmedin Jemal. 2016. "Breast Cancer Statistics, 2015: Convergence of Incidence Rates between Black and White Women." CA: A Cancer Journal for Clinicians 66 (1): 31-42.

Einav, Liran, Amy Finkelstein, and Heidi Williams. 2016. "Paying on the Margin for Medical Care: Evidence from Breast Cancer Treatments." American Economic Journal: Economic Policy 8 (1): 52-79.

Fan, Lei, Paul E. Goss, and Kathrin Strasser-Weippl. 2015. "Current Status and Future Projections of Breast Cancer in Asia." Breast Care (Basel) 10 (6): 373-78.

Garrison Jr., Louis P., Deborah Lubeck, Deepa Lalla, Virginia Paton, Amylou Dueck, and Edith A. Perez. 2007. "Cost-Effectiveness Analysis of Trastuzumab in the Adjuvant Setting for Treatment for HER2-positive Breast Cancer." Cancer 110 (3): 489-98.

Health Promotion Administration, Ministry of Health and Welfare. 2016. Cancer Registry Annual Report, 2013. Taipei, Taiwan: Ministry of Health and Welfare.

Hicks, Ian, Lucy Liu, and Linda Zhao. 2011. "The Challenges of Cancer Treatment in China." China Business Review, Jan. 1. Accessed Mar. 6, 2018. https://www .chinabusinessreview.com/the-challenges-of-cancer-treatment-in-china/.

Ho, M. L., Y. H. Hsiao, S. Y. Su, M. C. Chou, and Y. P. Liaw. 2015. "Mortality of Breast Cancer in Taiwan, 1971-2010: Temporal Changes and an Age-PeriodCohort Analysis.” Journal of Obstetrics and Gynaecology 35 (1): 60-63. 
Huang, C. S., C. H. Lin, Y. S. Lu, and C. Y. Shen. 2010. "Unique Features of Breast Cancer in Asian Women - Breast Cancer in Taiwan as an Example." Journal of Steroid Biochemistry and Molecular Biology 118 (4-5): 300-303.

Jourdan, Adam. 2017. "China Adds Blockbuster Drugs to Insurance List after Price Cuts.” Reuters News, July 18. Accessed Mar. 6, 2018. https://www.reuters.com /article/us-china-pharmaceuticals/china-adds-blockbuster-drugs-to-insurance -list-after-price-cuts-idUSKBN1A408F.

Kakwani, Nanak, Adam Wagstaff, and Eddy van Doorslaer. 1997. "Socioeconomic Inequalities in Health: Measurement, Computation and Statistical Inference." Journal of Econometrics 77 (1): 87-104.

Layne, T., J. Aminawung, P. Soulos, M. Nunez-Smith, and M. Nunez, B. Jones, K. Wang, and C. Gross. 2018. "Quality of Breast Cancer Care in the U.S. Territories: Insights from Medicare.” Health Affairs 37 (3): 421-28.

Li, J., B. Zhang, J. Fan, Y. Pang, P. Zhang, S. Wang, S. Zheng, et al. 2011. "A NationWide Multicenter 10-Year (1999-2008) Retrospective Clinical Epidemiological Study of Female Breast Cancer in China." BMC Cancer 11 (1) 364. http://doi.org /10.1186/1471-2407-11-364.

Lien, H. M. 2011. "How to Construct Social-Economic Variables from National Health Insurance Data.” Journal of Social Sciences and Philosophy 23 (3): 371-98.

Lin M. H., A. C. Yang, and T. H. Wen. 2011. "Using Regional Differences and Demographic Characteristics to Evaluate the Principles of Estimation of the Residence of the Population in National Health Insurance Research Databases (NHIRD)." Taiwan Journal of Public Health 30 (4): 347-61.

Lin S. J. 2008. "Factors Influencing the Uptake of Screening Services for Breast and Cervical Cancer in Taiwan." Journal of the Royal Society for the Promotion of Health 128 (6): 327-34.

Loibl, Sibylle, and Luca Gianni. 2017. "HER2-positive Breast Cancer.” Lancet 389 (10087): 2415-29.

Lu, Jui-fen Rachel, and Tung-Liang Chang. 2011. "Evolution of Taiwan's Health Care System." Health Economics, Policy and Law 6 (1): 85-107.

Lu, Jui-fen Rachel, Karen Eggleston, and Joseph Tung-Chieh Chang. 2018. "Economic Dimensions of Personalized and Precision Medicine in Asia: Evidence from Breast Cancer Treatment in Taiwan." Working Paper no. 48, Stanford Asia Health Policy Program. http://aparc.fsi.stanford.edu/asiahealthpolicy/research lasia_health_policy_program_working_paper_series.

Lu, Jui-fen Rachel, and William C. Hsiao. 2003. "Does Universal Health Insurance Make Health Care Unaffordable? Lessons from Taiwan.” Health Affairs 22 (3): 77-88.

Ministry of Health and Welfare. 2017. "National Health Expenditures, 2015." Accessed Oct. 21. http://www.mohw.gov.tw/lp-3537-2.html.

Neumann, Peter J., Theodore G. Ganiats, Louise B. Russell, Gillian D. Sanders, and Joanna E. Siegel, eds. 2017. Cost Effectiveness in Health and Medicine. New York: Oxford University Press.

Norton, Edward C., and Bryan E. Dowd. 2017. "Log Odds and the Interpretation of Logit Models." Health Services Research http://www.doi.org/10.1111/1475-6773 .12712.

Pan, Huay-Ben, Kam-Fai Wong, Tsung-Lung Yang, Giu-Cheng Hsu, Chen-Pin Chou, Jer-Shyung Huang, San-Kan Lee, Yi-Hong Chou, Chia-Ling Chiang, and Huei-Lung Liang. 2014. "The Outcome of a Quality-Controlled Mammography Screening Program: Experience from a Population-Based Study in Taiwan." Journal of the Chinese Medical Association 77 (10): 531-34.

Parkins, D. M., P. Pisani, and J. Ferlay. 1993. "Estimates of the Worldwide Inci- 
dence of Eighteen Major Cancers in 1985." International Journal of Cancer 54 (4): 594-606.

Pauly, Mark V. 2017. "Cost Sharing in Insurance Coverage for Precision Medicine." Draft for NBER conference, Sept. 2017.

Perez, Edith A., Edward H. Romond, Vera J. Suman, Jong-Hyeon Jeong, George Sledge, Charles E. Geyer Jr., Silvana Martino, et al. 2014. "Trastuzumab plus Adjuvant Chemotherapy for Human Epidermal Growth Factor Receptor 2-positive Breast Cancer: Planned Joint Analysis of Overall Survival from NSABP B-31 and NCCTG N9831." Journal of Clinical Oncology 32 (33): 3744-52.

Romond, Edward H., Edith A. Perez, John Bryant, Vera J. Suman, Charles E. Geyer Jr., Nancy E. Davidson, Elizabeth Tan-Chiu, et al. 2005. "Trastuzumab Plus Adjuvant Chemotherapy for Operable HER2-positive Breast Cancer." New England Journal of Medicine 353 (16): 1673-84.

Shen, Ying-Chun, Chee-Jen Chang, Chiun Hsu, Chia-Chi Cheng, Chang-Fang Chiu, and Ann-Lii Cheng. 2005. "Significant Difference in the Trends of Female Breast Cancer Incidence between Taiwanese and Caucasian Americans: Implications from Age-Period-Cohort Analysis." Cancer Epidemiology, Biomarkers, \& Prevention 14 (8): 1986-90.

Silva, A., Y. Molina, B. Hunt, T. Markossian, and N. Saiyed. 2017. "Potential Impact of the Affordable Care Act's Preventive Services Provision on Breast Cancer Stage: A Preliminary Assessment." Cancer Epidemiology 49:108-11. http://doi.org/10 .1016/j.canep.2017.05.015.

Slamon, Dennis, Wolfgang Eiermann, Nicholas Robert, Tadeusz Pienkowski, Miguel Martin, Michael Press, John Mackey, et al. 2011. "Adjuvant Trastuzumab in HER2-positive Breast Cancer." New England Journal of Medicine 365:1273-83.

Sohn, Ji-young. 2016. "Celltrion Seeks EMA Approval of Herceptin Biosimilar." Korea Herald, Oct. 31. Accessed Mar. 6, 2018. http://www.koreaherald.com/view .php?ud=20161031000895.

. 2017. "Celltrion Wins Herceptin Patent Trials against Roche in Korea." Korea Herald, Apr. 26. Accessed Mar. 6, 2018. http://www.koreaherald.com/view .php?ud=20170426001054.

Taiwan Cancer Registry (TCR). 2013. "Cancer Incidence Rate in Taiwan, 19982013.” Breast Cancer Five-Year Survival Rate. http://tcr.cph.ntu.edu.tw/main.php ?Page $=\mathrm{N} 2$.

Tarazi, W., C. Bradley, H. Bear, D. Harless, and L. Sabik. 2017. "Impact of Medicaid Disenrollment in Tennessee on Breast Cancer Stage at Diagnosis and Treatment." Cancer 123 (17): 3312-19. http://doi.org/10.1002/cncr.30771.

Tsuchida, Junko, Masayuki Nagahashi, Omar M. Rashid, Kazuaki Takabe, and Toshifumi Wakai. 2015. "At What Age Should Screening Mammography Be Recommended for Asian Women?" Cancer Medicine 4 (7): 1136-44.

Wang, C. Jason, Skye H. Cheng, and Jen-You Wu. 2017. "Association of a BundledPayment Program with Cost and Outcomes in Full-Cycle Breast Cancer Care." Journal of the American Medical Association: Oncology 3 (3): 327-34.

Wang, Qiong, Jing Li, Jia-Yuan Li, Yi Pang, Rong Huang, Bao-Ning Zhang, Bin Zhang, et al. 2012. "Breast Cancer Stage at Diagnosis and Area-Based Socioeconomic Status: A Multicenter 10-Year Retrospective Clinical Epidemiological Study in China." BMC Cancer 12:122.

World Health Organization (WHO). 2012. "Breast Cancer Estimated Incidence, Mortality and Prevalence Worldwide in 2012." WHO GLOBOCAN. Accessed Mar. 8, 2018. http://globocan.iarc.fr/old/FactSheets/cancers/breast-new.asp. 